\title{
1-D Periodic Lattice Sums for Complex and Leaky Waves in 2-D Structures Using Higher-Order Ewald Formulation
}

\author{
Vakhtang Jandieri, Senior Member, IEEE, Paolo Baccarelli, Member, IEEE, \\ Guido Valerio, Member, IEEE, and Giuseppe Schettini, Senior Member, IEEE
}

\begin{abstract}
A very efficient and accurate method is proposed to evaluate the Lattice Sums (LSs) for the analysis of leaky waves in two-dimensional (2-D) periodic waveguides. The LSs are series involving Hankel functions of arbitrary order, which are not convergent for complex wavenumbers. It is shown that by extending Ewald representations to higher-order Hankel functions, the LSs can be expressed in terms of spatial and spectral series, granting Gaussian convergence even in the case of complex and leaky waves. The method allows for the appropriate choice of the spectral determination for each space harmonic of a given LS coefficient, thus permitting one to obtain modal solutions that may correspond to physical and non-physical leakywave phenomena. First, the proposed LS calculation is exploited in the evaluation of the free-space one-dimensional (1-D) periodic Green's function for 2-D structures. Then, the same procedure for the LSs is implemented in a cylindrical harmonic expansion method, based on the transition-matrix and the generalized reflection-matrix approach, for the full-wave analysis of leaky modes in 2-D Electromagnetic Band-Gap waveguides formed by layered arrays of cylindrical inclusions. The presented LS formalism is numerically slim, very fast, and thus well suited for the analysis of a significant class of lossy periodic waveguides and leaky-wave antennas.
\end{abstract}

Index Terms - Leaky waves, complex waves, modal analysis, periodic structures, Green's functions, electromagnetic band-gap materials, photonic crystals.

\section{INTRODUCTION}

$\mathrm{P}$ ERIODIC dielectric or metallic structures have been a subject of continuous interest for applications to frequency or polarization-selective devices from microwaves to optical waves [1]. One-dimensional (1-D) periodic arrays of infinitely long parallel strips or cylinders embedded in dielectric structures are typical kinds of discrete periodic

V. Jandieri is with General and Theoretical Electrical Engineering (ATE), Faculty of Engineering, University of Duisburg-Essen, and CENIDE - Center for Nanointegration Duisburg-Essen, D-47048 Duisburg, Germany (e-mail: vakhtang.jandieri@uni-due.de).

P. Baccarelli and G. Schettini are with "Roma Tre" University, EMLAB Laboratory of Electromagnetic Fields, Department of Engineering, Via Vito Volterra 62, 00146 Rome, Italy, and CNIT, "Roma Tre" Unit (e-mail: paolo.baccarelli@uniroma3.it; giuseppe.schettini@uniroma3.it).

G. Valerio is with Sorbonne Université, Laboratoire d'Electronique et Electromagnetisme, 75252, Paris, France (e-mail: guido.valerio@sorbonneuniversite.fr) systems. These structures are classified as two-dimensional (2D) since they are geometrically invariant in the direction of infinite extension, i.e., $z$-direction in Fig. 1, along which also the fields are assumed to not vary. When the array is multilayered, it gives rise to 2-D Frequency Selective Structures (FSSs) and Electromagnetic Band-Gap (EBG) structures in which electromagnetic filtering is achieved and wave propagation is allowed or forbidden within a possibly large frequency range [2], [3], [4]. Furthermore, defect waveguides can be obtained by removing one or a few rows of rods in EBG structures to achieve low-loss propagation in the millimeterwave and optical ranges [5], [6] as well as highly directive radiation [7]-[9].

Most of the numerical methods available for the full-wave analysis of 2-D electromagnetic scattering by 1-D periodic gratings are based on integral-equation formulation and require the computation of the free-space periodic Green's function (PGF), typically expressed as an infinite series of zero-th order Hankel functions. Different spectral and integral approaches have been used for the fast computation of the PGF in 2-D problems with 1-D periodicity [10]-[18]. Some of the proposed methods are based on an alternative representation of the 1-D PGF that, by applying Graf's addition theorem for the Hankel functions, leads to a Neumann series expansion whose coefficients are named Lattice Sums (LSs) [19]-[23]. The LS method becomes particularly effective when the PGF needs to be evaluated many times [19]. Furthermore, effective and tailored methods for the electromagnetic modeling of 2-D photonic crystals composed by cylindrical inclusion are based on the cylindrical-wave expansion (CWE) in conjunction with either the transition-matrix (T-matrix) approach [24]-[27] or the Rayleigh's method and mode-matching [28]-[32]. In these cases, the reflection and transmission matrices, which relate the incident space-harmonics to the reflected and transmitted ones, are inherently expressed in terms of LSs.

The LSs are a class of Schlömilch series whose terms are expressed as a combination of trigonometric and Hankel functions [33]. The LSs in the original spatial form converge very slowly and are unsuitable for numerical calculation [34]. Different representations have been proposed for accelerating the numerical convergence of the LSs, from the seminal work of Twersky [34], where expressions amenable for numerical computation have been provided, to the improved formulations proposed in [22], [19]-[21], and in [35]. Accelerated 
computation of the LSs are based on either suitable integral identities or Ewald representations, as reported in [23], [35] and [36]-[38], respectively. In all the proposed formulations, a pure real phase shift among adjacent cells is considered in the quasi-periodic (i.e., Bloch) problem, and hence in the evaluation of the LSs. This case is of typical interest in the general context of the electromagnetic scattering by periodic gratings. However, there are particular interesting applications of the PGFs and of the relevant LS coefficients where a complex Bloch wavenumber is needed. Typical cases are the modal analyses in periodic gratings, where complex wavenumbers are obtained because of radiative losses (i.e., leaky modes) or ohmic and dielectric losses of the media forming the periodic waveguides. Also, modes in stopband regions are characterized by complex wavenumbers. The knowledge of the modal complex propagation wavenumber is of significant importance for the investigation of the leakage phenomena and the analysis of the radiation features in EBG waveguides and antennas [39], [40], for the design and fabrication of novel leaky-wave antennas [41], [42], and for the studies of a wide class of waveguide-based passive filters with dielectric and ohmic losses [43], [44]. We finally note that complex wavenumbers are also needed if the LSs are used in full-wave procedures to calculate the near field excited by a non-periodic line source on infinite 1-D periodic 2-D gratings [45]-[47]. Unfortunately, the spatial expression of the LSs [27], [34], [37] is not convergent for complex Bloch wavenumbers and careful attention needs to be paid in order to extend the computation to such a case.

In this paper, highly convergent representations of the LSs are applied to the specific case of general complex wavenumber. The method is originated from the standard Ewald transformation [48]. Since LSs involve Hankel functions of any integer order $m$, the Ewald representation extended to the $m$-th order Hankel function is suitably used for the accurate and efficient evaluation of each LS, which has been then split into spectral and spatial Ewald series. The Gaussian asymptotic behavior of such higher-order Ewald series involved in the LSs grants a fast convergence, even in the presence of complex wavenumbers. Furthermore, the proper and improper nature of the field, which is fundamental to correctly assess the leakage phenomena [41], can be taken into account by suitably determining the transverse field behavior of each space harmonic in the relevant spectral Ewald series. Some preliminary results on the capability of the present method to calculate the LSs in the case of complex propagation wavenumbers have been shown in [49]-[51], which are considerably extended here by providing all the details of the analytical derivation and reporting a complete set of results and validations.

The complex-mode dispersive behaviors in 2-D FSSs or EBG waveguides can be analyzed by using either generalpurpose periodic mixed-potential integral-equation formulations in conjunction with the method of moments (MoM) [52], [53], or hybrid techniques which combine the finite element (FEM) or full boundary element (BEM) approaches with the periodic spectral-domain Green's function of layered media [54]-[56], or the Fourier Series Expansion

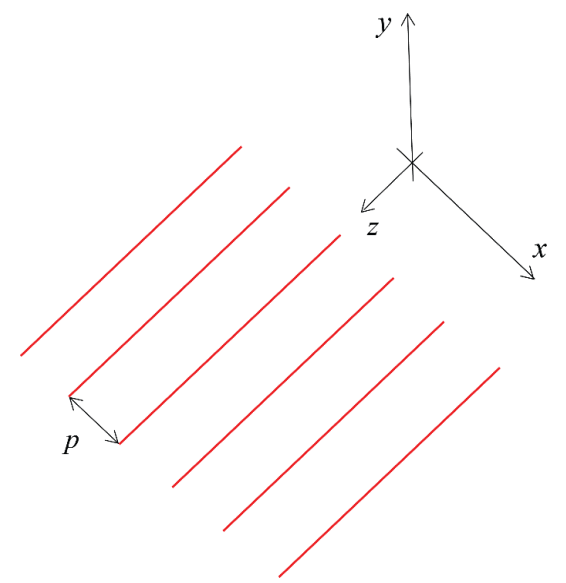

(a)

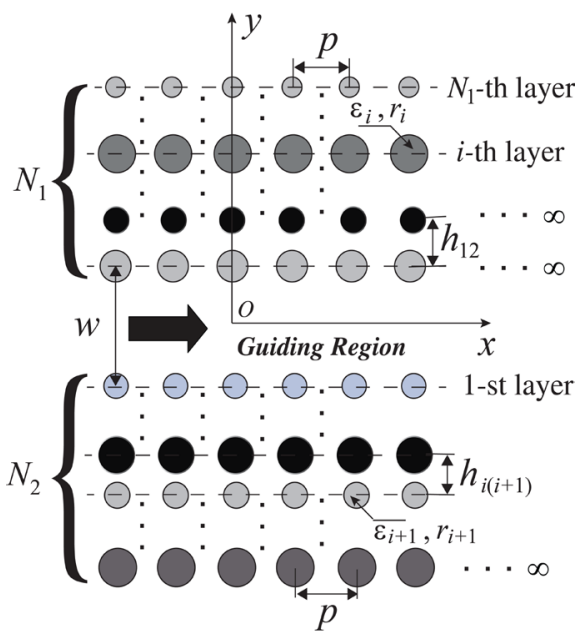

(b)

Fig. 1. (a) 1-D array of line sources in free-space with period $p$ along the $x$ axis (the problem is independent of the $z$ coordinate). (b) Side view of a 2-D EBG waveguide sandwiched by two EBGs composed of $N_{1}$ and $N_{2}$ number of layers, each layer being a 1-D array of circular rods in free space having a common period $p$. Distance between the adjacent $i$-th and $(i+1)$-th periodic layers is $h_{i(i+1)}$, dielectric permittivity and the radius of the rods on the $i$-th layer are $\varepsilon i$ and $r_{i}$, respectively and the width of the EBG waveguide is $w$.

method (FSEM) combined with perfectly matched layers (PMLs) [57], or the Dirichlet-to-Neumann (DtN) map method [58]. Here, the developed procedure for the efficient computation of the LSs with complex wavenumbers is implemented in a FORTRAN numerical code. It is then applied to two independent canonical electromagnetic problems, which can be profitably used for the study of guided complex and leaky modes: $i$ ) The evaluation of the free-space 1-D PGF for 2-D structures; ii) The full-wave modeling of EBG waveguides by means of cylindrical harmonic expansion methods.

The former problem is applicable to general numerical methods based on integral-equation formulations [52], [53], whereas the latter is particularly suited to the study of structures composed by 1-D periodic arrays of infinitely long parallel cylinders [1, Ch. 3]. In both cases, results obtained with the proposed approach are validated by means of wellestablished independent methods. The new procedure for the calculation of the LSs is shown to be accurate, robust, and 
extremely fast, thus representing an effective numerical tool for the full-wave analysis of 2-D structures with 1-D periodicity in the presence of complex wavenumbers.

This paper is organized as follows. In Section II, the numerical evaluation of the free-space 1-D PGF for 2-D structures and the full-wave modal analysis of 2-D EBG waveguides is formulated in terms of LSs. A discussion of the advantages of using the LS approach and of the divergence of the LS series in the presence of a complex wavenumber is provided. In Section III, the accelerated computation of the LSs using higher-order Ewald representations is illustrated in detail. Spatial and spectral Ewald series are suitably derived and the convergence properties of the obtained expressions are investigated. Finally, the possibility of considering the proper and improper nature of each space harmonic composing the spectral Ewald series is described. In Section IV, numerical results for the accelerated 1-D PGF, evaluated for different choices of complex wavenumbers and kinds of modal regimes, and the leaky-mode dispersion properties of EBG structures are shown and compared with independent results, as a validation of the proposed approach.

\section{Formulation OF THE PROBLEM AND THEORETICAL BACKGROUND}

In this section, the LS formulation is described in the context of two independent electromagnetic problems. First, the calculation of the 2-D PGF of an array of line sources is considered, which can be suitably used in full-wave numerical approaches based on integral equations discretized with the MoM. In this framework, the PGF needs to be typically calculated for numerous combinations of source and observation points, thus making the LS approach particularly effective and suitable.

Then, a full-wave method based on the cylindrical harmonic expansion is examined. This approach is particularly suitable for the analysis of 2-D EBG structures formed by array of circular cylinders, where the effective representation of the scattered fields from periodic objects naturally involves the LS formulation, but does not require the computation of a PGF. In all cases the occurrence of complex Bloch wavenumbers is considered. A time-harmonic dependence $e^{j \omega t}$ is assumed and suppressed throughout this paper.

\section{A. 2-D Green's function with 1-D Periodicity and the Lattice Sums.}

The Green's function of a 1-D array of line sources that are parallel to the $z$ axis and periodically spaced along the direction $x$ (see Fig. 1(a)) in a homogeneous medium, which without loss of generality we take to be the free-space, is given by the following expressions [14], [23]:

$$
\begin{gathered}
G(x, y)=\frac{1}{4 j} \sum_{n=-\infty}^{+\infty} H_{0}^{(2)}\left[k_{0} \sqrt{(x-n p)^{2}+y^{2}}\right] e^{-j n k_{x 0} p} \\
=\frac{1}{2 j p} \sum_{n=-\infty}^{+\infty} \frac{e^{-j \sqrt{k_{0}^{2}-k_{x n}^{2}}|y|}}{\sqrt{k_{0}^{2}-k_{x n}^{2}}} e^{-j k_{x x} x}
\end{gathered}
$$

The first series in (1) is a spatial-domain representation, where $H_{0}^{(2)}$ is the zero-th order Hankel function of the second kind, $k_{x 0}$ the propagation wavenumber (or the phase shift), $k_{0}$ is the free-space wavenumber, and $p$ is the period. The second series in (1) is a spectral-domain expression based on Poisson's formula [14], [15], where the field is represented as a superposition of an infinite number of space harmonics. The $n$-th space harmonic has an $x$-dependence of the kind $e^{-j k_{x x} x}$, with a complex wavenumber $k_{x n}=k_{x 0}+2 \pi n / p$. As is known, the spatial series in (1) is not convergent in the general case of a complex wavenumber $k_{x 0}=\beta_{0}-j \alpha$, with $\beta_{0}$ and $\alpha$ the relevant phase and attenuation constants, since the $e^{-n \alpha p}$ contribution is exponentially diverging with either increasing positive or negative $n$ (depending on the sign of $\alpha$ ) while the Hankel function decreases with a rate slower than exponential [14]. On the other hand, the spectral series in (1) or the integral representation using Ewald transformation (see Appendix A, [13], [14]) can be suitably applied for complex wavenumbers. In particular, the latter yields a very fast (Gaussian) converging behavior even in the worst-case scenario of on-plane calculation (i.e., $y=0$ ), where the former converges only algebraically.

An alternative way to calculate the Green's function has been proposed in [19]-[23], which benefits from a Neumann series representation of the spatial series in (1). First, the series in (1) can be rewritten as follows:

$$
\begin{aligned}
& \frac{1}{4 j} \sum_{n=-\infty}^{+\infty} H_{0}^{(2)}\left(k_{0} \rho_{n}\right) e^{-j n k_{x 0} p}=\frac{1}{4 j} H_{0}^{(2)}\left(k_{0} \rho\right) \\
& +\frac{1}{4 j}\left[\sum_{n=1}^{+\infty} H_{0}^{(2)}\left(k_{0} \rho_{n}\right) e^{-j n k_{x} p}+\sum_{n=1}^{+\infty} H_{0}^{(2)}\left(k_{0} \rho_{-n}\right) e^{j n k_{x 0} p}\right]
\end{aligned}
$$

where $\rho_{n}=\left[(x-n p)^{2}+y^{2}\right]^{1 / 2}$ and $\rho=\rho_{0}$. Then, by removing one of the line sources, a cylindrical region of radius $p$ centered about the vacated line source is source free and the field at an arbitrary observation point $\rho$ in the region can be expanded in a series of Bessel functions. In fact, within the domain defined by $\rho<p$, we may apply the Graf's addition theorem for the Hankel functions [59]:

$$
\begin{aligned}
& H_{0}^{(2)}\left(k_{0} \rho_{n}\right)=\sum_{m=-\infty}^{+\infty} H_{m}^{(2)}\left(k_{0} n p\right) J_{m}\left(k_{0} \rho\right) e^{-j m \theta} \\
& H_{0}^{(2)}\left(k_{0} \rho_{-n}\right)=\sum_{m=-\infty}^{+\infty}(-1)^{m} H_{m}^{(2)}\left(k_{0} n p\right) J_{m}\left(k_{0} \rho\right) e^{-j m \theta}
\end{aligned}
$$

where $\theta=\cos ^{-1}(x / \rho)$. Hence, we expand the series within brackets in (1) in terms of Bessel functions [19]-[23]:

$$
\begin{aligned}
& G(x, y)=\frac{1}{4 j}\left[H_{0}^{(2)}\left(k_{0} \rho\right)+L_{0}\left(k_{x 0} p, k_{0} p\right) J_{0}\left(k_{0} \rho\right)\right. \\
& \left.+2 \sum_{m=1}^{+\infty} L_{m}\left(k_{x 0} p, k_{0} p\right) J_{m}\left(k_{0} \rho\right) \cos (m \theta)\right]
\end{aligned}
$$

where $J_{m}$ is the Bessel function of the first kind and $m$-th order and $L_{m}$ is an expansion coefficient called the Lattice Sum 
(LS). The coefficients defined by the LS are expressed in the following form, as an infinite sum over multipole line sources [19]-[23]:

$$
L_{m}\left(k_{x 0} p, k_{0} p\right)=\sum_{n=1}^{+\infty} H_{m}^{(2)}\left(k_{0} n p\right)\left[e^{-j n k_{x 0} p}+(-1)^{m} e^{j n k_{x 0} p}\right]
$$

for non-negative $m$.

We observe that every LS coefficient is independent of the observation points and this can represent a significant computational advantage when the PGF needs to be evaluated many times, since we need to calculate LSs only once. Unfortunately, each LS in (5) is very slowly convergent for real wavenumbers $k_{x 0}=\beta_{0}$ and diverges for complex wavenumbers $k_{x 0}=\beta_{0}-j \alpha$. In Section III a highly convergent expression for the LSs will be applied to the case of complex wavenumbers. This expression is based on higher-order spectral and spatial Ewald series, and it easily accounts for the proper and improper determination of the modal fields [41]. The computation time for the evaluation of the 1-D PGF using the proposed approach for the calculation of the LSs will be shown to be lower than that based on the standard application of the Ewald decomposition [13], [14], as the number of the observation points increases within the unit cell.

\section{B. Modal Analysis of 2-D EBG Structures using the Cylindrical-wave Expansion, the Transition Matrix, and the Lattice Sums.}

The full-wave modal analysis of EBG structures formed by an array of circular cylinders can be performed with a MoM [53] making use of the computation of the PGF as described in the previous subsection. An alternative method is briefly described in this subsection; it does not require the direct calculation of a PGF, but it is still based on the LS technique [25]. This full-wave method [1, Ch. 3], [27] exploits the effectiveness of the T-matrix approach, which characterizes the nature of the scatterer, and of the generalized reflection and transmission matrices of the layered periodic structure, which can be evaluated by using an effective recursive algorithm [60]. Furthermore, LSs only characterize the periodic arrangements of the scatterers and are independent of the polarization, observations points, and geometrical parameters of the scatterers [19]-[23], thus representing a very smart approach. However, in order to investigate leakage phenomena, a rapidly convergent formulation for the calculation of the LSs with complex wavenumbers is needed, as also commented in [27].

The side view of a 2-D EBG waveguide is shown in Fig. 1(b). The upper and the lower EBG structures consist of $N_{1}$ and $N_{2}$ layered arrays of dielectric circular cylinders, which are infinitely long in the $z$ direction and periodically spaced with a common distance $p$ in the $x$ direction. The cylindrical elements should be the same along each layer of the arrays, but those in different layers can be different both in geometrical and material properties. The permittivity and radius of the rods along the $i$-th periodic layer are $\varepsilon_{i}$ and $r_{i}$, respectively, and the distance between the adjacent $i$-th and $(i+1)$-th layers is $h_{i(i+1)}$. The background medium is homogeneous, and is assumed to be the free space. The guiding region is formed by removing one or few rows of the rods from the original lattice, thus resulting in a multimode EBG waveguide having a width $w$. The leaky modal field propagating in the 1-D periodic waveguide can be represented as a superposition of an infinite number of space harmonics. The $n$-th space harmonic has an $x$ dependence of the kind $e^{-j k_{x n} x}$, with a complex wavenumber $k_{x n}=k_{x 0}+2 \pi n / p$ and $k_{x 0}=\beta_{0}-j \alpha$. Hence, each space harmonic propagates along the $x$ axis with a different phase constant $\beta_{n}=\beta_{0}+2 \pi n / p$, but attenuates with the same leakage constant $\alpha$.

When the array is multilayered as shown in Fig. 1(b), the scattered space harmonics impinge on the neighbor arrays as new incident waves and are scattered into another set of space harmonics, which then impinges back on the original array. A concatenation of these processes explains the multiple scattering of wave fields in the layered arrays. The scattering process from each layer of the structure is characterized by the reflection and transmission matrices, which relate a set of the incident space harmonics to a set of reflected and transmitted ones. Due to symmetry reasons, the incident and scattered modal fields related to each cylinder within the unit cell can be expressed as sets of appropriate cylindrical waves [1], [25], [39], [60]. Reflection $\mathbf{R}_{i}$ and transmission $\mathbf{F}_{i}$ matrices for the arrays located on $y=y_{i}$ are given as follows [25]:

$\mathbf{R}_{i}=\mathbf{U}^{+}\left(k_{x 0}\right)\left[\mathbf{I}-\mathbf{T}\left(k_{0}\right) \mathbf{L}\left(k_{x 0} p, k_{0} p\right)\right]^{-1} \mathbf{T}\left(k_{0}\right) \mathbf{P}\left(k_{x 0}\right)$

$\mathbf{F}_{i}=\mathbf{I}+\mathbf{U}^{-}\left(k_{x 0}\right)\left[\mathbf{I}-\mathbf{T}\left(k_{x 0}\right) \mathbf{L}\left(k_{x 0} p, k_{0} p\right)\right]^{-1} \mathbf{T}\left(k_{0}\right) \mathbf{P}\left(k_{x 0}\right)$

with

$$
\begin{aligned}
& \mathbf{P}\left(k_{x 0}\right)=\left[P_{s q}\left(k_{x 0}\right)\right]=\left[(-j)^{s} e^{-j s \cos ^{-1}\left(k_{x q} / k_{0}\right)}\right] \\
& \mathbf{U}^{ \pm}\left(k_{x 0}\right)=\left[U_{n s}^{ \pm}\left(k_{x 0}\right)\right]=\left[\frac{2 j^{s}}{k_{y n} p} e^{\mp j s \cos ^{-1}\left(k_{x n} / k_{0}\right)}\right] \\
& \mathbf{L}\left(k_{x 0} p, k_{0} p\right)=\left[L_{q-s}\left(k_{x 0} p, k_{0} p\right)\right]
\end{aligned}
$$

$\mathbf{P}\left(k_{x 0}\right)$ is a matrix that transforms the down-going $q$-th incident space harmonic wave to the $s$-th cylindrical harmonic wave. $\mathbf{U}^{+}$ and $\mathbf{U}^{-}$are the matrices transforming the $s$-th cylindrical harmonic wave back to the up-going and down-going $n$-th space-harmonic waves (with $n, s, q=-M,-M+1, \ldots, 0, \ldots, M$ $-1, M)$. The $n$-th row and $q$-th column elements of the reflection $\mathbf{R}_{i}=\left[R_{i}^{n q}\right]$ and transmission $\mathbf{F}_{i}=\left[F_{i}^{n q}\right]$ matrices represent the reflection and transmission coefficients of the $n$ th order reflected and transmitted space harmonics for the $q$-th order space harmonic of the incident wave. Because of the isotropic nature of the scatterers, the reflection and transmission matrices for both incident down-going and upgoing space harmonics are the same and are equal to $\mathbf{R}_{i}=\left[R_{i}^{n q}\right]$ and $\mathbf{F}_{i}=\left[F_{i}^{n q}\right]$, respectively. The transverse wavenumber of the $n$-th space-harmonic is $k_{y n}=\left(k_{0}^{2}-k_{x n}^{2}\right)^{1 / 2}$, I is the unit matrix, $\mathbf{T}\left(k_{0}\right)$ is the T-matrix of the isolated circular rod [25] describing the nature of the scattered field per unit cell, and $\mathbf{L}\left(k_{x 0} p, k_{0} p\right)$ is the matrix of the LSs. Note that all the matrices in (6) - (9) are given as functions of the complex wavenumber $k_{x 0}$, whereas the T-matrix does not depend on $k_{x 0}$, 
but only on the geometrical and physical parameters of the single cylinder and on the free-space wavenumber $k_{0}$.

The matrix $\mathbf{L}\left(k_{x 0} p, k_{0} p\right)$ defined in (10), whose elements can be expressed as in (5), is a key element of the present formulation. Hence, also in this case a highly convergent and effective numerical procedure for the evaluation of the LSs with complex Bloch wavenumbers is needed to apply the present method to guided complex and leaky modes. The details of the derivation will be shown in Section III.

The complex Bloch wavenumber $k_{x 0}$ can be found solving the following dispersion equation:

$$
\operatorname{det}\left[\mathbf{I}-\mathbf{D}\left(k_{x 0}\right) \cdot \overline{\overline{\mathbf{R}}}\left(k_{x 0}\right) \cdot \mathbf{D}\left(k_{x 0}\right) \cdot \overline{\overline{\mathbf{R}}}\left(k_{x 0}\right)\right]=0
$$

with

$$
\mathbf{D}=\left[\exp \left(-j k_{y n} w\right)\right]
$$

where $\mathbf{D}\left(k_{x 0}\right)$ represents the transverse traveling wave behavior of the space harmonics in the guiding region having a width $w$ and $\overline{\overline{\mathbf{R}}}\left(k_{x 0}\right)$ is the generalized reflection matrix for the layered periodic structure array viewed from the innermost region (guiding region in Fig. 1(b)). The latter can be easily obtained based on a recursive algorithm [25], [60], [61] using (6) and (7). The calculation process is very fast since only simple matrix multiplications are involved.

It should be mentioned that the formulation based on LSs technique is very general and flexible, since it can be applied to the full-wave analysis of leaky modes in periodic and bandgap structures composed of any kind of layered periodic arrays of dielectric circular rods [1] or of plasmonic crystals or periodic chains formed by metal nanocylinders [62]. Furthermore, the method can also consider background medium characterized by a tensor permeability [63] and the formalism can be extended to scatterers with non-circular cylindrical cross section, by numerically deriving the relevant T-matrix expression [64].

\section{Calculation of The LatTice SuMS USING THE EWALD TRANSFORMATION}

In this section, an effective technique for the computation of the series (5) in the presence of complex wavenumbers is presented, based on higher-order representations of the standard Ewald transformation for the 1-D PGF of phased line sources [13], [14].

From expressions (1)-(4) for the 1-D PGF we can write:

$$
\sum_{n=-\infty}^{+\infty} H_{0}^{(2)}\left(k_{0} \rho_{n}\right) e^{-j n k_{x 0} p}=H_{0}^{(2)}\left(k_{0} \rho\right)+\sum_{m=-\infty}^{+\infty} J_{m}\left(k_{0} \rho\right) e^{-j m \theta} L_{m}
$$

where $L_{m}$ is the $m$-th LS in (5). Using the standard Ewald transformation reported in Appendix A, (13) can be cast in the following form:

$$
\begin{aligned}
& \sum_{m=-\infty}^{\infty} J_{m}\left(k_{0} \rho\right) e^{-j m \theta} L_{m}=-H_{0}^{(2)}\left(k_{0} \rho\right)+\frac{2 j}{\pi} \\
& \times\left(\int_{E_{s p l}}^{\infty} d \eta \frac{e^{-\frac{\rho^{2} \eta^{2}}{p^{2}}+\frac{k_{0}^{2} p^{2}}{4 \eta^{2}}}}{\eta}+\sum_{\substack{n=-\infty \\
n \neq 0}}^{+\infty} e^{-j n k_{x 0} p} \int_{E_{s p l}}^{\infty} d \eta \frac{e^{-\frac{\rho_{n}^{2} \eta^{2}}{p^{2}}+\frac{k_{0}^{2} p^{2}}{4 \eta^{2}}}}{\eta}\right) \\
& +\frac{2 j}{\pi} \sum_{n=-\infty}^{\infty} e^{-j n k_{x 0} p} \int_{0}^{E_{s p l}} d \eta \frac{e^{-\frac{\rho_{n}^{2} \eta^{2}}{p^{2}}+\frac{k_{0}^{2} p^{2}}{4 \eta^{2}}}}{\eta}
\end{aligned}
$$

where the Ewald splitting parameter $E_{s p l}$ that gives the best possible convergence of the overall spatial and spectral series combination should be chosen as for the standard Ewald decomposition in [65]. Multiplying both sides of (14) by $e^{j m^{\prime} \theta}$, integrating in the $\theta$ variable from 0 to $2 \pi$ and taking into account that:

$$
\begin{aligned}
& \int_{0}^{2 \pi} e^{j\left(m^{\prime}-m\right) \theta} d \theta=2 \pi \delta_{m m^{\prime}} \\
& \int_{0}^{2 \pi} \sum_{m=-\infty}^{\infty} J_{m}\left(k_{0} \rho\right) L_{m} e^{j\left(m^{\prime}-m\right) \theta} d \theta=2 \pi J_{m^{\prime}}\left(k_{0} \rho\right) L_{m^{\prime}}
\end{aligned}
$$

with $\rho_{n}=\left[(\rho \cos \theta-n p)^{2}+\rho^{2} \sin ^{2} \theta\right]^{1 / 2}$ and $\delta_{m m^{\prime}}$ the Kronecker delta function, we obtain the following:

$$
\begin{aligned}
& J_{m^{\prime}}\left(k_{0} \rho\right) L_{m^{\prime}}= \\
& -\delta_{m^{\prime} 0}\left(H_{0}^{(2)}\left(k_{0} \rho\right)-\frac{2 j}{\pi} \int_{E_{s p l}}^{\infty} \frac{e^{-\frac{\rho^{2} \eta^{2}}{p^{2}}+\frac{k_{0}^{2} p^{2}}{4 \eta^{2}}}}{\eta} d \eta\right) \\
& +\frac{j}{\pi^{2}} \sum_{n=-\infty}^{+\infty} e^{-j n k_{x 0} p} \int_{E_{s p l}}^{\infty} \frac{e^{\frac{k_{0}^{2} p^{2}}{4 \eta^{2}}}}{\eta}\left(\int_{0}^{2 \pi} e^{-\frac{\rho_{n}^{2} \eta^{2}}{p^{2}}} e^{j m^{\prime} \theta} d \theta\right) d \eta \\
& +\frac{j}{\pi^{2}} \sum_{n=-\infty}^{+\infty} e^{-j n k_{x 0} p} \int_{0}^{E_{s p l}} \frac{e^{\frac{k_{0}^{2} p^{2}}{4 \eta^{2}}}}{\eta}\left(\int_{0}^{2 \pi} e^{-\frac{\rho_{n}^{2} \eta^{2}}{p^{2}}} e^{j m^{\prime} \theta} d \theta\right) d \eta
\end{aligned}
$$

Since the LSs are independent of $\rho$, we can obtain $L_{m^{\prime}}$ from (16) dividing the left and right sides by $J_{m^{\prime}}\left(k_{0} \rho\right)$ and taking the limit $\rho \rightarrow 0$. Furthermore, we observe that the first and second terms the right hand of (16) have the form of a $m$-th order spatial Ewald series, whereas the third term will be cast as a $m$-th order spectral Ewald series. Hence, we can write the $m$-th LS in terms of $m$-th order spectral and spatial Ewald series:

$L_{m}\left(k_{x 0} p, k_{0} p\right)=L_{m}^{\mathrm{spec}}\left(k_{x 0} p, k_{0} p\right)+L_{m}^{\mathrm{spat}}\left(k_{x 0} p, k_{0} p\right)$

where 


$$
\begin{aligned}
& L_{m \geq 0}^{\mathrm{spec}}\left(k_{x 0} p, k_{0} p\right)=\frac{j}{\pi^{2}} \lim _{\rho \rightarrow 0}\left[\frac{1}{J_{m}\left(k_{0} \rho\right)}\right. \\
& \left.\times \sum_{n=-\infty}^{+\infty} e^{-j n k_{x 0} p} \int_{0}^{E_{s p l}} \frac{e^{\frac{k_{0}^{2} p^{2}}{4 \eta^{2}}}}{\eta}\left(\int_{0}^{2 \pi} e^{-\frac{\rho_{n}^{2} \eta^{2}}{p^{2}}} e^{j m \theta} d \theta\right) d \eta\right]
\end{aligned}
$$

and

$$
\begin{aligned}
& L_{m \geq 0}^{\mathrm{spat}}\left(k_{x 0} p, k_{0} p\right)= \\
& \lim _{\rho \rightarrow 0}\left[-\delta_{m 0}\left(H_{0}^{(2)}\left(k_{0} \rho\right)-\frac{2 j}{\pi} \int_{E_{s p l}}^{\infty} \frac{e^{-\frac{\rho^{2} \eta^{2}}{p^{2}}+\frac{k_{0}^{2} p^{2}}{4 \eta^{2}}}}{\eta} d \eta\right)\right. \\
& \left.+\frac{j}{\pi^{2} J_{m}\left(k_{0} \rho\right)} \sum_{\substack{n=-\infty \\
n \neq 0}}^{+\infty} e^{-j n k_{x 0} p} \int_{E_{s p l}}^{\infty} \frac{e^{\frac{k_{0}^{2} p^{2}}{4 \eta^{2}}}}{\eta}\left(\int_{0}^{2 \pi} e^{-\frac{\rho_{n}^{2} \eta^{2}}{p^{2}}} e^{j m \theta} d \theta\right) d \eta\right]
\end{aligned}
$$

For the first term on the right side of (19) we considered that $J_{0}\left(k_{0} \rho\right) \rightarrow 1$ when $\rho \rightarrow 0$. Furthermore, the LS elements with the negative index $m$, can be simply calculated as $L_{-m}=$ $(-1)^{m} L_{m}$.

\section{A. Spatial Ewald Series of the Lattice Sums.}

In this subsection, we derive the expression for the $m$-th order spatial Ewald series of $L_{m}$. The cases $m \neq 0$ and $m=0$ are considered separately. For $m \neq 0$, the first term in (19) vanishes and the spatial Ewald series of the LS is reduced to the following expression:

$$
\begin{aligned}
& L_{m>0}^{\mathrm{spat}}=\lim _{\rho \rightarrow 0}\left[\frac{j}{\pi^{2} J_{m}\left(k_{0} \rho\right)}\right. \\
& \left.\times \sum_{\substack{n=-\infty \\
n \neq 0}}^{+\infty} e^{-j n k_{x 0} p} \int_{E_{s p l}}^{\infty} \frac{e^{\frac{k_{0}^{2} p^{2}}{4 \eta^{2}}}}{\eta}\left(\int_{0}^{2 \pi} e^{-\frac{\rho_{n}^{2} \eta^{2}}{p^{2}}} e^{j m \theta} d \theta\right) d \eta\right]
\end{aligned}
$$

where

$$
\int_{0}^{2 \pi} e^{-\frac{\rho_{n}^{2} \eta^{2}}{p^{2}}} e^{j m \theta} d \theta=2 \pi e^{-\frac{\rho^{2} \eta^{2}}{p^{2}}-n^{2} \eta^{2}} I_{m}\left(2 \eta^{2} n \rho / p\right)
$$

with $I_{m}$ the modified Bessel function of the first kind [59]. Since we need the limit $\rho \rightarrow 0$, we use the following small argument approximations for the Bessel and modified Bessel function of the first kind [66]:

$$
\begin{aligned}
& J_{m}\left(k_{0} \rho\right) \approx\left(k_{0} \rho / 2\right)^{m} / \Gamma(m+1) \\
& I_{m}\left(2 \eta^{2} n \rho / p\right) \approx\left(\eta^{2} n \rho / p\right)^{m} / \Gamma(m+1),
\end{aligned}
$$

respectively, where $\Gamma$ is the gamma function [59]. Finally, (20) can be written as follows:

$$
\begin{aligned}
& L_{m>0}^{\mathrm{spat}}=-\frac{2^{m+1}}{j \pi} \sum_{\substack{n=-\infty \\
n \neq 0}}^{+\infty} e^{-j n k_{x 0} p}\left(\frac{n}{k_{0} p}\right)^{m} Q_{m}= \\
& -\frac{2^{m+1}}{j \pi} \sum_{n=1}^{+\infty}\left[e^{-j n k_{x 0} p}+(-1)^{m} e^{j n k_{x 0} p}\right]\left(\frac{n}{k_{0} p}\right)^{m} Q_{m}
\end{aligned}
$$

with

$$
Q_{m}=\int_{E_{s p l}}^{\infty} \frac{e^{\frac{k_{0}^{2} p^{2}}{4 \eta^{2}}} e^{-n^{2} \eta^{2}}}{\eta^{-2 m+1}} d \eta .
$$

We note that $Q_{m}$ is an even function with respect to $n$ and has a Gaussian convergence with $n$. The latter property assures an effective convergence of the $m$-th order spatial Ewald series (24) for complex wavenumbers, i.e., $k_{x 0}=\beta_{0}-j \alpha$.

The integral in (25) can be suitably calculated using either the trapezoidal or Simpson's rule [67]. However, in order to significantly speed-up the calculation of this integral, we apply the following recurrence relation [68]:

$$
Q_{m+1}=\frac{1}{2 n^{2}}\left(2 m Q_{m}-\frac{k_{0}^{2} p^{2}}{2} Q_{m-1}+E_{s p l}^{2 m} e^{-n^{2} E_{s p l}^{2}} e^{k_{0}^{2} p^{2} / 4 E_{s p l}^{2}}\right)
$$

In this case, we need only to calculate integrals $Q_{0}$ and $Q_{1}$, which can be easily expressed through the exponential integral $E_{q}(z)$ in the following form:

$$
\begin{aligned}
& Q_{0}=\frac{1}{2} \sum_{s=0}^{\infty}\left(\frac{k_{0} p}{2 E_{s p l}}\right)^{2 p} \frac{1}{s !} E_{s+1}\left(n^{2} E_{s p l}^{2}\right) \\
& Q_{1}=\frac{E_{s p l}^{2}}{2}\left[\frac{1}{n^{2} E_{s p l}^{2}} e^{-n^{2} E_{s p l}^{2}}+\sum_{s=1}^{+\infty}\left(\frac{k_{0} p}{2}\right)^{2 p} \frac{1}{s !} \frac{1}{E_{s p l}^{2 s}} E_{s}\left(n^{2} E_{s p l}^{2}\right)\right]
\end{aligned}
$$

Integrals $Q_{m}(m \geq 2)$ can then be easily calculated based on the recurrence relation (26).

For the case $m=0$, considering (19), (27), and the expressions (49) derived in Appendix B, $L_{0}^{\text {spat }}$ can be written as follows:

$$
L_{0}^{\mathrm{spat}}=-1+\frac{j}{\pi} \mathrm{Ei}\left(\frac{k_{0}^{2} p^{2}}{4 E_{s p l}^{2}}\right)-\frac{4}{j \pi} \sum_{n=1}^{+\infty} \cos \left(n k_{x 0} p\right) Q_{0}
$$

where again the Gaussian convergence with $n$ of $Q_{0}$ allows for the evaluation of (29) when $k_{x 0}=\beta_{0}-j \alpha$; Ei is an exponential integral.

We finally note that, by letting $E_{s p l} \rightarrow 0,(18)$ vanishes and the LS coefficients in (5) are entirely described by (24), (25), and (29), thus allowing for retrieving the Ewald transformation extended to higher-order Hankel functions (see [66], Section 8.42 , and [69]), which in the general case $\rho \neq 0$ assumes the following expression 


$$
H_{m}^{(2)}\left(k_{0}, \rho_{n}\right)=\frac{2^{m+1}}{-j \pi}\left(\frac{\rho_{n}}{k_{0} p^{2}}\right)^{m} \int_{0}^{\infty} d \eta \frac{e^{-\frac{\rho_{n}^{2} \eta^{2}}{p^{2}}+\frac{k_{0}^{2} p^{2}}{4 \eta^{2}}}}{\eta^{1-2 m}}
$$

with the standard Ewald transformation in (38) being the zeroth order case.

\section{B. Spectral Ewald Series of the Lattice Sums.}

Let us rewrite the $m$-th order spectral Ewald series (18) in the form:

$$
\begin{aligned}
& L_{m \geq 0}^{\mathrm{spec}}=\frac{j}{\pi^{2}} \lim _{\rho \rightarrow 0}\left[\frac{1}{J_{m}\left(k_{0} \rho\right)}\right. \\
& \left.\times \int_{0}^{2 \pi}\left(\int_{0}^{E_{s p l}} \frac{e^{-\frac{y^{2} \eta^{2}}{p^{2}}+\left(\frac{k_{0} p}{2 \eta}\right)^{2}}}{\eta} \Phi(x) d \eta\right) e^{j m \theta} d \theta\right]
\end{aligned}
$$

where $\Phi(x)$ is the following Floquet periodic function:

$$
\Phi(x)=\sum_{n=-\infty}^{+\infty} \phi(x-n p) e^{-j n k_{x 0} p}
$$

with $\phi(x-n p)=\exp \left[-(x-n p)^{2} \eta^{2} / p^{2}\right]$. By applying Poisson's formula [14], $\Phi(x)$ can be given in terms of an infinite superposition of space harmonics, which are waves with complex propagation wavenumbers expressed as $k_{x n}=k_{x 0}+2 \pi n / p$, with $k_{x 0}=\beta_{0}-j \alpha$ and $n=0, \pm 1, \pm 2, \ldots$. Hence, $\Phi(x)$ has the following form:

$\Phi(x)=\frac{1}{p} \sum_{n=-\infty}^{+\infty} g\left(\rho k_{x n}\right) e^{-j k_{x n} x}$

where the following Fourier transform expression is used:

$g\left(\rho k_{x n}\right)=\int_{-\infty}^{+\infty} \phi(x) e^{j k_{x n} x} d x=\frac{p \sqrt{\pi}}{\eta} e^{-\left(\frac{k_{x n} p}{2 \eta}\right)^{2}}$.

Substituting (32)-(34) in (31) we obtain:

$$
\begin{aligned}
& L_{m \geq 0}^{\text {spec }}=\frac{j}{\pi^{3 / 2}} \lim _{\rho \rightarrow 0}\left[\frac{1}{J_{m}\left(k_{0} \rho\right)}\right. \\
& \left.\times \int_{0}^{2 \pi}\left(\sum_{n=-\infty}^{+\infty} e^{-j k_{x n} x} \int_{0}^{E_{s p l}} \frac{e^{-\frac{y^{2} \eta^{2}}{p^{2}}+\frac{k_{y n}^{2} p^{2}}{4 \eta^{2}}}}{\eta^{2}} d \eta\right) e^{j m \theta} d \theta\right]
\end{aligned}
$$

where $k_{y n}=\left(k_{0}^{2}-k_{x n}^{2}\right)^{1 / 2}$ is the transverse wavenumber of the $n$-th space harmonic.

Following the calculation procedure presented in Appendix $\mathrm{C}$ and considering (57) and (58), we can write the spectral Ewald series in the following form:

$$
\begin{aligned}
& L_{m \geq 0}^{\mathrm{spec}}=\frac{2(-j)^{m}}{p} \\
& \times \sum_{n=-\infty}^{+\infty}\left(\frac{k_{x n}}{k_{0}}\right)^{m} \sum_{s^{\prime}=0}^{[m / 2]}(-1)^{s^{\prime}}\left(\begin{array}{c}
m \\
2 s^{\prime}
\end{array}\right)\left(\frac{k_{y n}}{k_{x n}}\right)^{2 s^{\prime}} C_{s^{\prime}, n}
\end{aligned}
$$

where

$$
C_{s^{\prime}, n}=\frac{1}{k_{y n}} \operatorname{erfc}\left(j \frac{p k_{y n}}{2 E_{s p l}}\right)-\frac{e^{\left(\frac{p k_{y n}}{2 E_{s p l}}\right)^{2}}}{k_{y n}} \sum_{i=1}^{s^{\prime}} \frac{\left(j \frac{p k_{y n}}{2 E_{s p l}}\right)^{1-2 i}}{\Gamma\left(\frac{3}{2}-j\right)}
$$

with $[\mathrm{m} / 2]$ defined in (56) and the complementary error function (erfc) as in [59]. We note that the convergence of the space-harmonic expansion in (36) is ensured by the Gaussian behavior of $C_{s^{\prime}, n}$ with increasing $|n|$, also in the presence of complex wavenumbers $k_{x n}=k_{x 0}+2 \pi n / p$, with $k_{x 0}=\beta_{0}-j \alpha$ and $n=0, \pm 1, \pm 2, \ldots$. Furthermore, the determination of the square root defining the transverse wavenumber of the $n$-th space harmonic, i.e., $k_{y n}=\left(k_{0}^{2}-k_{x n}^{2}\right)^{1 / 2}$, in the terms (37) of the $m$-th order spectral Ewald series (36), is chosen according to the specific problem one is solving. In particular, if $\mathfrak{I m}\left\{k_{y n}\right\} \leq 0$ the $n$-th harmonic is said to be proper. If instead $\mathfrak{I m}\left\{k_{y n}\right\}>0$, the $n$-th harmonic is improper. In modal problems, only a finite number of harmonics may be improper (leaky modes), thus ensuring the convergence of (36) for each $m$.

\section{NUMERICAL RESULTS AND DISCUSSIONS}

In this section, numerical results for several different kinds of problems are shown that validate the LSs calculation described in this paper. This effective LS formulation is implemented in a computer code and used in two independent environments. In particular, the accuracy, convergence behavior, and efficiency of the approach proposed to calculate the 1-D PGF for 2-D structures are illustrated in Section IV-A and the relevant results are compared and validated with those obtained by means of an independent and well-established standard Ewald approach [13], [14]. Furthermore, a reference full-wave method [1, Ch.3] has been extended to the rigorous analysis of the dispersion behavior of the leaky-mode phase and attenuation constants of EBG waveguides. In Section IV$\mathrm{B}$, real and imaginary parts of the propagation wavenumber are calculated and compared with those obtained by means of the PMLs-FSEM in [57] and the DtN map method in [58], thus fully validating the proposed approach.

\section{A. Green's function computation with LSS}

The fast converging expressions for the LSs proposed in the previous section allow for the computation of the PGF by means of the series (4). In Fig. 2 we calculate the Green's function with a standard Ewald approach (granting a Gaussian convergence) and the LSs expression (4), whose convergence is discussed in the following. Three different cases of complex wavenumbers are considered: A backward leaky wave (all the harmonics are chosen with a proper determination), and two 


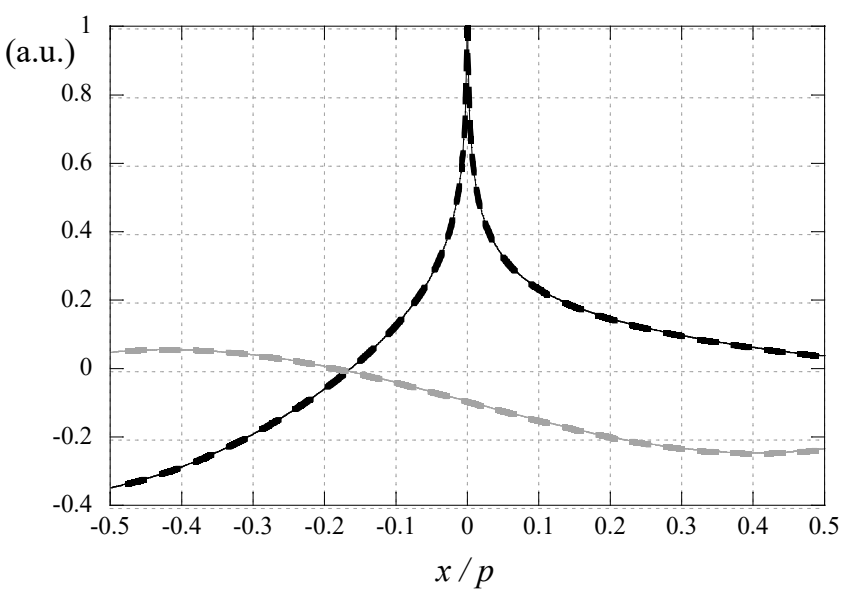

(a)

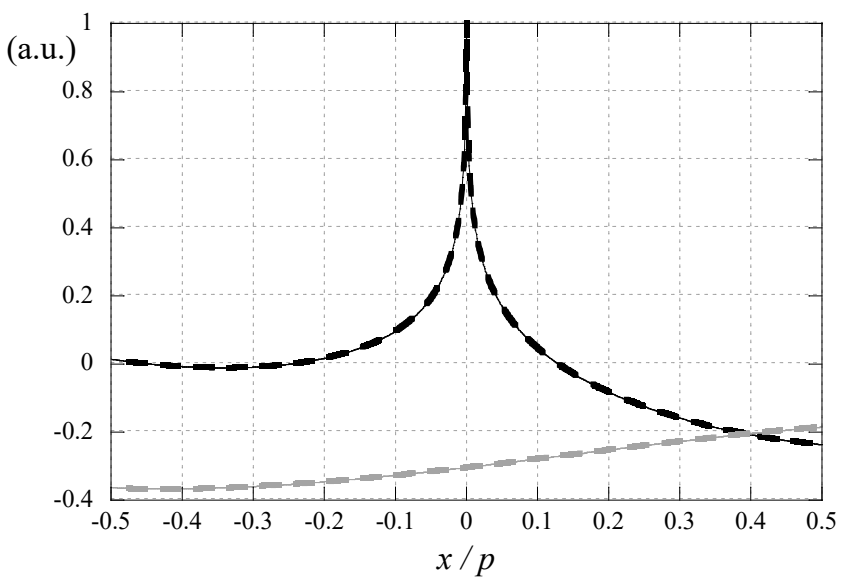

(b)

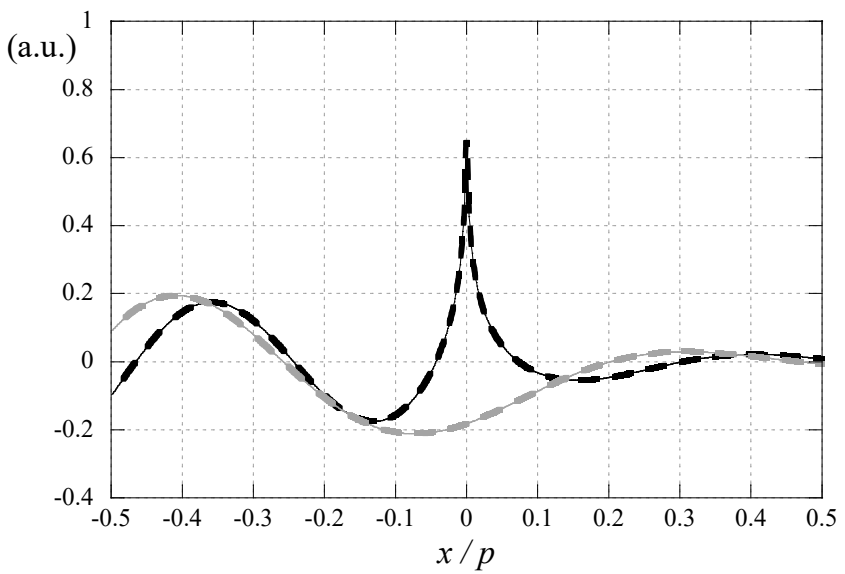

(c)

Fig. 2. Green's functions of a 1-D array of line sources parallel to the $z$ axis and periodically located along the direction $x$ (as shown Fig. 1(a)) computed with the standard Ewald method (solid curves) and LSs series (4) (dashed curves). Real parts (black curves) and imaginary parts (gray curves). Frequency $f=10 \mathrm{GHz}$. (a) Complex wavenumber $k_{x 0}=(-0.5-j 0.1) k_{0}$ (all the harmonics have a proper determination; the $n=0$ harmonic is fast) and period $p=0.6 \lambda$. (b) Complex wavenumber $k_{x 0}=(\lambda / p+0.5-j 0.1) k_{0}$ (the harmonic $n=-1$ is fast and has an improper determination) and period $p=$ $0.3 \lambda$. (c) Complex wavenumber $k_{x 0}=(-0.25-j 0.2) k_{0}$ (the harmonics $n=0$ and $n=1$ are fast and have an improper determination) and period $p=2 \lambda$. The $y$-axis label (a.u.) stands for arbitrary unit.

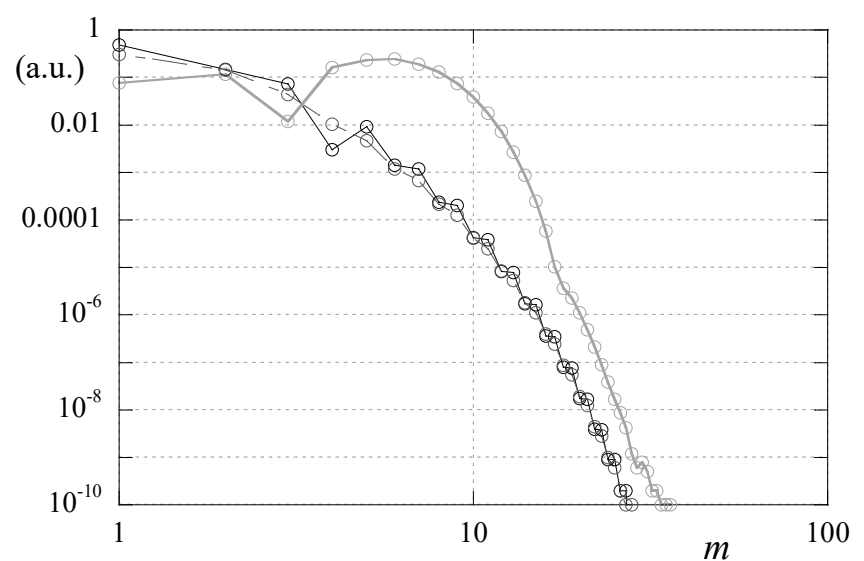

Fig. 3. Decay of the magnitude of the terms $\left|L_{m}\left(k_{x 0} p, k_{0} p\right) J_{m}\left(k_{0} \rho\right)\right|$ in (4) versus the summation index $m$, for $\rho=p / 2$ and $y=0$ (on-plane case). Parameters as in Fig. 2(a) (black curve), in Fig. 2(b) (dark gray curve), and in Fig. 2(c) (light gray curve). The $y$-axis label (a.u.) stands for arbitrary unit.

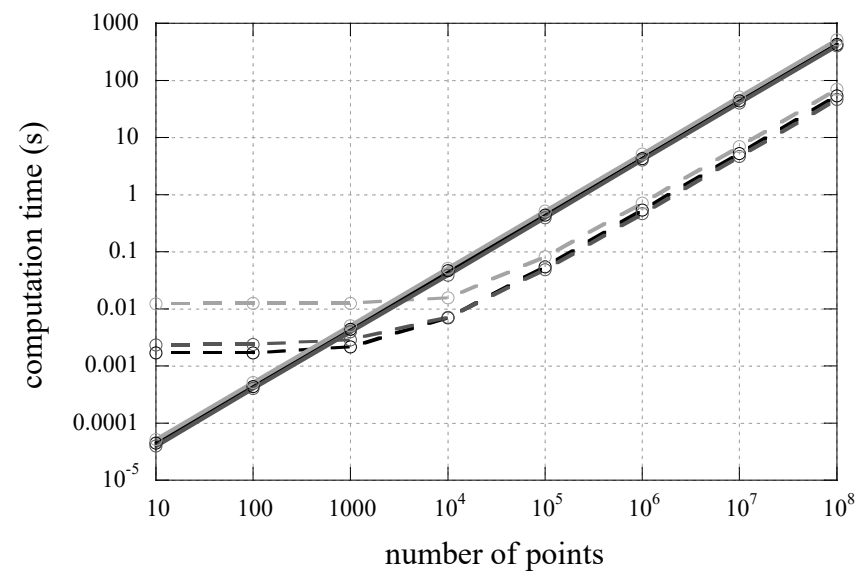

Fig. 4. Time in seconds for the computation of the Green's function in an equi-spaced number of points in the interval $-p / 2 \leq x \leq p / 2, y=0$ (onplane case). Two computation methods are compared: Ewald decomposition (solid lines) and LSs series (dashed lines). Parameters as in Fig. 2(a) (black curve), in Fig. 2(b) (dark gray curve), and in Fig. 2(c) (light gray curve).

forward leaky waves (the first with one harmonic having an improper determination, the second with two harmonics having an improper determination). The comparisons of the results show a very good agreement between the two methods, thus validating the LS approach for complex wavenumbers, with proper and improper harmonics.

The LS coefficients have a Gaussian convergence and need to be computed only once, even if the Green's function is needed at several points, since they are not coordinates dependent. This strategy is then expected to become faster than the simple Ewald method when the number of samples required is sufficiently large.

This is true if the $m$-series in (4) converges sufficiently fast. It is easy to realize that this is the case. In fact, (4) can be regarded as Fourier series of the Green's function with respect to the variable $\theta$, in the domain $\rho<p$ due to the Graf theorem hypothesis used to obtain (3). The $m$-series is smooth with respect to $\theta$ in this domain: in fact, the only singularity of the 


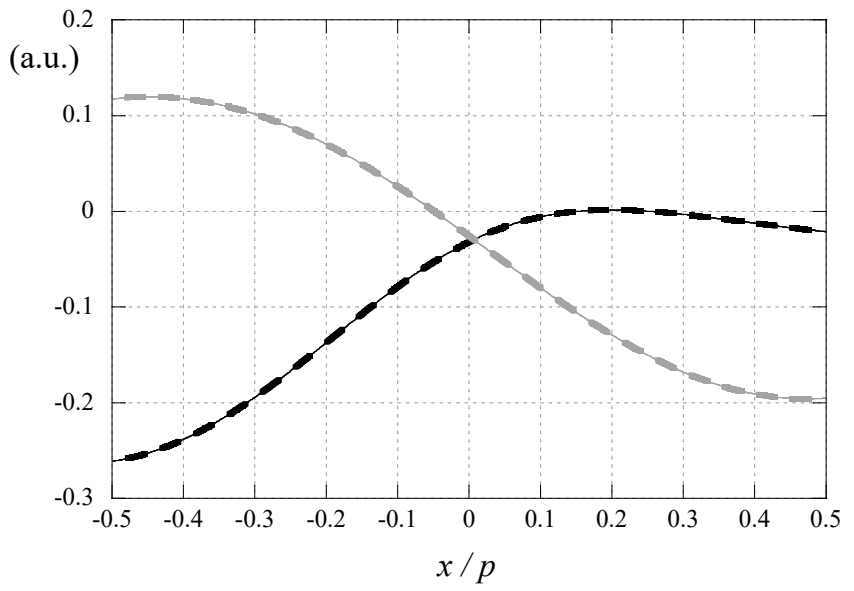

(a)

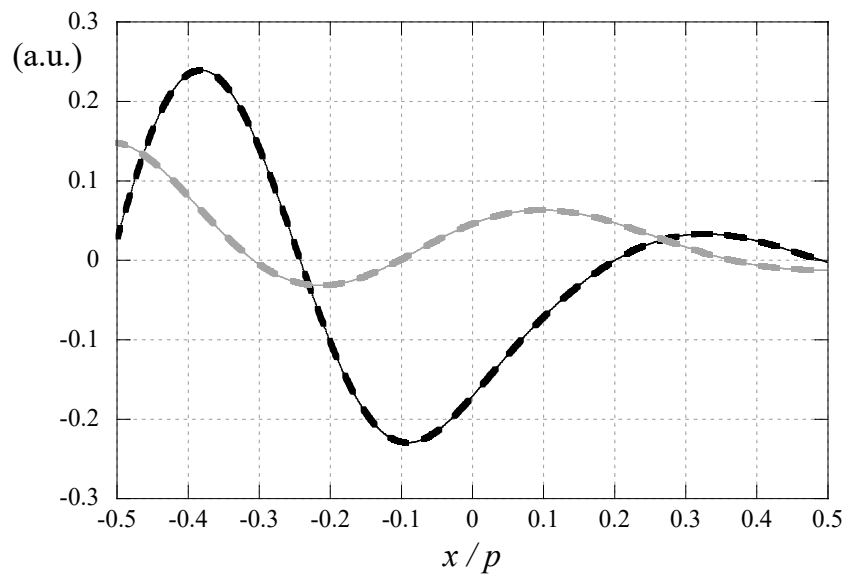

(b)

Fig. 5. Periodic Green's functions computed with the standard Ewald method (solid curves) and LSs series (4) (dashed curves). Real parts (black curves) and imaginary parts (gray curves). Frequency $f=10 \mathrm{GHz}$. (a) Complex wavenumber $k_{x 0}=(-0.5-j 0.1) k_{0}$ (all the harmonics have a proper determination; the $n=0$ harmonic is fast), period $p=0.6 \lambda, y=0.334 p$. (b) Complex wavenumber $k_{x 0}=(-0.25-j 0.2) k_{0}$ (the harmonics $n=0$ and $n=1$ are fast and have an improper determination) and period $p=2 \lambda, y=0.167 p$. The y-axis label (a.u.) stands for arbitrary unit.

Green's function in this domain, at $\rho=0$, has been fully extracted from the series as a separate term (the first one) of (4). This smoothness grants to the series a faster-than-algebraic converge rate according to Fourier-series theory [70]. In Fig. 3 , the decay of the magnitude of the $\left|L_{m}\left(k_{x 0} p, k_{0} p\right) J_{m}\left(k_{0} \rho\right)\right|$ terms of (4) with respect to the summation index $m$ is shown at

$\rho=p / 2$ for the three cases considered in Fig. 2. A Gaussian convergence is observed in all the cases, thus confirming the expected behavior.

In Fig. 4, the computation time in seconds spent to calculate the three previous Green's functions is shown, with an accuracy of $10^{-4}$, and over an increasing number of sample points. As expected, the LS-based computation becomes faster than the Ewald method for more than $10^{3}-10^{4}$ samples. The tests have been performed on an Intel Xeon CPU X5650 @ $2.67 \mathrm{GHz}$ with $96 \mathrm{GHz}$ of RAM.

All the computation tests have been here performed in the most critical on-plane case $(y=0)$, where the spectral series in
(1) converges only algebraically; by contrast, both the standard Ewald approach and the proposed LS formulation have Gaussian convergence. Since the LS expressions are still valid as long as $\rho<p$, we also compared the LS formulation (4) with the standard Ewald approach for the off-plane case $(y \neq$ 0 ). The results for two different cases of complex wavenumbers are shown in Fig. 5. Excellent agreement between the two methods is still observed. Finally, we note that outside the domain of validity of the LS formulation, i.e., $\rho \geq p$, the simple spectral series in (1) is assumed sufficiently fast-converging (exponential) to be used instead of both the LS series or the Ewald series [14].

In order to further speed up the calculation, we note that the PGF can be properly regularized, by removing near-singular terms at the edges of the unit cell, thus also allowing for a more efficient interpolation. Suitable regularization and interpolation procedures have been presented in the literature, which can also be adapted to the present derivation [71], [72].

\section{B. Leaky modes in pillar type (WI) EBG Waveguide.}

The method described in this manuscript has been validated on the pillar type (W1) EBG waveguide. The EBG structure bounding the waveguide presents a square lattice (i.e., the distance $h$ between adjacent layers in Fig. 1(b) is the same and $h=p$ ), where all circular rods in Fig. 1(b) are chosen identical, with a radius $r=0.2 p$, and made of a lossless nonmagnetic material with dielectric permittivity $\varepsilon=11.90 \varepsilon_{0}$. The width of the waveguide is $w=2 h$. The results obtained for the TE modes with the current method have been compared with those from independent approaches based on the FSEM with PMLs [57] and the DtN maps [58]. In particular, we have independently developed the numerical code in [57] following the relevant formulation.

In Figs. 6 and 7, we show the dispersion behavior of the waveguide for different number of rows of cylinders: $N_{1}=N_{2}$ $=2$ and $N_{1}=N_{2}=3$, respectively. Analyses not reported here for the sake of brevity prove that for large $N_{1}$ and $N_{2}$ (in this case, $N_{1}=N_{2}=N>6$ ) the EBG structure bounding the waveguide shows a complete stopband region within the normalized frequency range $0.303<p / \lambda_{0}<0.432$, where $\lambda_{0}$ is the free-space wavelength [57]. Inside this band-gap region, the modal fields of the pillar-type waveguide are strongly confined, thus resulting in a purely real modal propagation wavenumber [57]. However, when the number of the layers of cylinders $N_{1}, N_{2}$ is decreased, the EBG structure loses its complete band-gap properties, the modal field of the W1 waveguide leaks out from the guiding structure, and the relevant wavenumber becomes complex. The case $N_{1}=N_{2}=2$ is treated in Fig. 6, where the normalized phase constant for the $n=0$ harmonic, $\beta_{0} p / 2 \pi$, and the modal normalized attenuation constant, $\alpha p / 2 \pi$, of the lowest TE leaky mode obtained by using LS technique are shown as a function of the normalized frequency $p / \lambda_{0}$. We note that in the reported frequency range only the $n=0$ space harmonic is fast, 


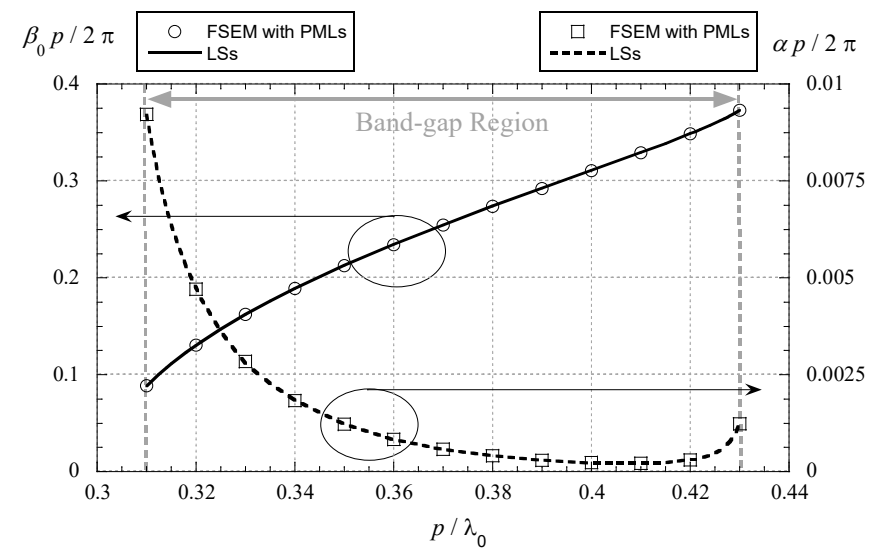

Fig. 6. Dispersion diagrams of the normalized phase $\beta_{0} p / 2 \pi$ and attenuation $\alpha p / 2 \pi$ constants for the lowest order TE leaky mode of a 2layered W1 EBG waveguide, obtained with the LSs technique (solid and dashed line, respectively) and FSEM with PML (black circles and square, respectively). The circular rods are characterized by the dielectric permittivity $\varepsilon=11.9 \varepsilon_{0}$ and the radius of rods is $r=0.2 p$.

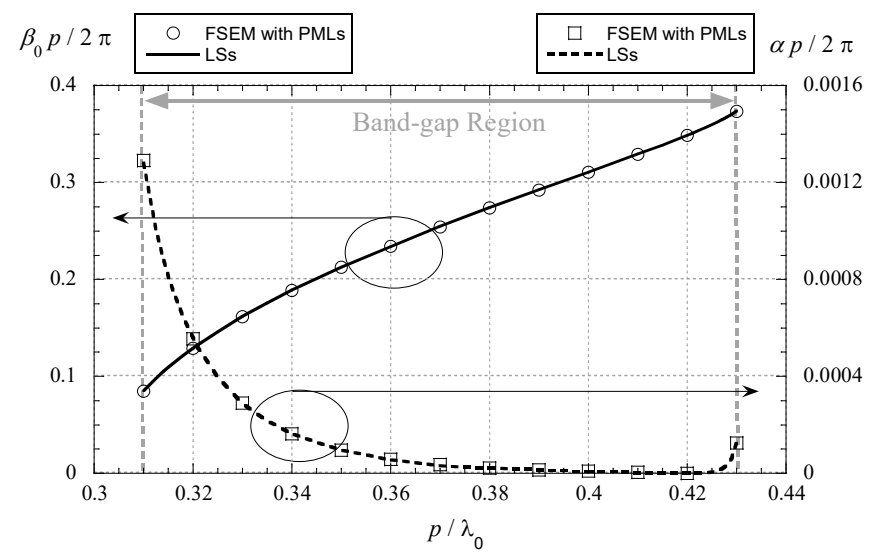

Fig. 7. The same as in Fig. 6 but for a 3-layered W1 EBG waveguide.

$\beta_{0}<2 \pi / \lambda_{0}$, and has an improper determination, i.e., $\operatorname{Im}\left\{k_{y 0}\right\}>0$, in (9), (12), and (37), whereas all other harmonics are proper [73]. The black circles and squares are obtained with the FSEM utilizing PMLs and show very good agreement with the LS approach. Furthermore, it should be noted that the modal attenuation constant becomes significant and rapidly increases as the wavelength approaches the band edges $p / \lambda_{0} \cong 0.303$ and $p / \lambda_{0} \cong 0.432$. This could be explained by a decrease of the field confinement in the EBG structure. In Fig. 7 the dispersion behavior of the same leaky mode as in Fig. 6 is shown, but for a three-layered structure, i.e., $N=3$. As expected, when the number of the EBG layers is increased, the mode confinement in the guiding region is stronger and, hence, the modal attenuation constant $\alpha p / 2 \pi$ substantially decreases. On the other hand, the phase constant, $\beta_{0} p / 2 \pi$, shows no noticeable change. It can be observed also in this case that the agreement between the present method based on the LSs and the FSEM with PMLs is very good.
Table 1 shows the convergence behavior of the normalized phase and attenuation constant versus the truncation number $M$ used in the reflection and transmission matrices in (6)-(9) to analyze the two-layered structure (as in Fig. 6) at $p / \lambda_{0}=0.35$ (the LSs have been evaluated with relative error $\left.10^{-6}\right)$. We note that $M$ is the truncation number of both the space- and cylindrical-harmonic expansions. The numerical analysis has shown that under the prescribed geometrical parameters the phase and attenuation constants do not change in the first 7 digits for $M \geq 7$ (totally 15 harmonics). Furthermore, our results agree with those in [57] (Fig. 7) up to the first three digits in the normalized phase constant and first four digits in the normalized attenuation constant and agrees with [58] (p. 982) up to the first 4 digits. We finally note that the proposed full-wave modal solver, based on the LS approach, is highly efficient and provides a significant speedup over standard procedures. In particular, the computation time to obtain the complex wavenumber for one frequency point and $M=7$ in Table I was approximately $0.07 \mathrm{~s}$, whereas that for the FSEM-PML as in [57] is around $25 \mathrm{~s}$. The tests have been performed on an Intel Core i7@3.6 GHz with 8 GB of RAM.

TABLE I

Phase AND ATtenuation CONSTANTS, $\beta_{0} p / 2 \pi$ AND $\alpha p / 2 \pi$,

RESPECTIVELY, VERSUS THE TRUNCATION NUMBER $M$ OF THE REFLECTION AND TRANSMISSION MATRICES IN (6)-(9) FOR THE TWO-LAYERED STRUCTURE (AS IN FIG. 6 ) AT $p / \lambda_{0}=0.35$ AND RELEVANT COMPUTATION TIMES (THE LS HAS BEEN EVALUATED WITH THE RELATIVE ERROR $10^{-6}$ ).

\begin{tabular}{|c|c|c|c|}
\hline \hline$M$ & $\beta_{0} p / 2 \pi$ & $\alpha p / 2 \pi$ & Time [sec] \\
\hline 1 & 0.2127300 & 0.0012272 & 0.066320 \\
\hline 3 & 0.2127890 & 0.0012120 & 0.067302 \\
\hline 5 & 0.2128410 & 0.0012200 & 0.067723 \\
\hline 7 & 0.2128620 & 0.0012256 & 0.067925 \\
\hline 9 & 0.2128620 & 0.0012256 & 0.068002 \\
\hline
\end{tabular}

\section{CONCLUSION}

The Lattice Sums (LSs), which are widely used as expansion coefficients of Neumann series in the evaluation of the free-space one-dimensional (1-D) periodic Green's function (PGF) of line sources and in the electromagnetic scattering from composite materials formed by periodic arrays of circular cylinders, have been extended to the analysis of guided complex and leaky waves. The extension of the evaluation of the LSs to the case of complex wavenumbers has been made possible thanks to the suitable representation of the semi-infinite sum of Hankel functions, which typically yields the LS coefficients in terms of higher-order spatial and spectral Ewald series. The proposed approach has shown a Gaussian convergence and allowed for the correct spectral determination of each spatial harmonic constituting the leaky modal field.

The enhanced LSs procedure has been applied to the evaluation of the 1-D PGF of line sources in a homogeneous medium and implemented in a full-wave numerical approach for the modal analysis of two-dimensional (2-D) EBG waveguides. In both cases, complex wavenumbers and proper 
and improper leaky-wave fields have been considered. The numerical procedures based on the LSs have been then validated by carefully comparing the relevant results with those obtained from independent approaches. Very good agreement has been observed between the different methods and, in all cases, the LSs showed improved efficiency.

The LS method is shown to be suitable for analyzing periodic leaky-wave structures and, in general, complex guided and leaky modes in typical 2-D planar periodic and EBG structures, including plasmonic crystals or periodic chains formed by metal nanocylinders. The proposed procedure can be easily applied to all the existing full-wave numerical approaches based on LSs when complex wavenumbers need to be treated.

\section{ACKNOWLEDGMENT}

V. Jandieri acknowledges financial support from the Alexander von Humboldt Foundation. P. Baccarelli and G. Schettini would like to acknowledge the Italian Ministry of Education (MIUR) for funding the Programma di Ricerca di Interesse Nazionale (PRIN 2015) project: Ultra-wideband Virtual Imaging Extra Wall (UView).

\section{APPENDIX A}

The Ewald decomposition [48] is used to express the PGF as a sum of two series, both having Gaussian convergence. Several decompositions are possible according to the number of periodicity directions and the dimension of the space. In the case of interest of this paper, i.e., a 1-D periodic array of line sources [13], [14], the Ewald transformation for 2-D cylindrical radiated fields is the following:

$$
H_{0}^{(2)}\left(k_{0}, \rho_{n}\right)=\frac{2}{-j \pi} \int_{0}^{\infty} d \eta \frac{e^{-\frac{\rho_{n}^{2} \eta^{2}}{p^{2}}+\frac{k_{0}^{2} p^{2}}{4 \eta^{2}}}}{\eta}
$$

where $\rho_{n}=\left[(x-n p)^{2}+y^{2}\right]^{1 / 2}$. Hence, by splitting the integral in (38) into two parts, the decomposition for the Green's function (1) reads as follows:

$$
G(x, y)=G^{\mathrm{spat}}(x, y)+G^{\mathrm{spec}}(x, y)
$$

with

$$
\begin{aligned}
& G^{\mathrm{spat}}(x, y)=\frac{1}{2 \pi} \sum_{n=-\infty}^{+\infty} e^{-j n k_{x 0} p} \int_{E_{s p l}}^{\infty} d \eta \frac{e^{-\frac{\rho_{n}^{2} \eta^{2}}{p^{2}}+\frac{k_{0}^{2} p^{2}}{4 \eta^{2}}}}{\eta} \\
& G^{\mathrm{spec}}(x, y)=\frac{1}{2 \pi} \sum_{n=-\infty}^{\infty} e^{-j n k_{x 0} p} \int_{0}^{E_{s p l}} d \eta \frac{e^{-\frac{\rho_{n}^{2} \eta^{2}}{p^{2}}+\frac{k_{0}^{2} p^{2}}{4 \eta^{2}}}}{\eta}
\end{aligned}
$$

The integrals in each term of (39) can be solved in closed form, leading to a Gaussian-converging series easy to calculate. The series in (41) can be transformed via the Poisson rule into a spectral series whose terms can be easily computed and whose convergence is also Gaussian. The value of the number $E_{s p l}$ can be arbitrarily chosen, but specific values have been derived [14], [65] in order to minimize the computation time by balancing the convergence of the two series by avoiding at the same time the high-frequency breakdown, i.e. cancellation errors between the series that occur at high frequencies. (However, the breakdown can still occur at sufficiently high frequencies when the period is equivalent to several wavelengths, but this case is not considered of interest for the structure investigated here).

\section{APPENDIX B}

When $m=0$ we need to evaluate the limit of the first term on the right side of (19) as follows:

$\lim _{\rho \rightarrow 0}\left(-H_{0}^{(2)}\left(k_{0} \rho\right)+\frac{2 j}{\pi} \int_{E_{s p l}}^{\infty} \frac{e^{-\frac{\rho^{2} \eta^{2}}{p^{2}}+\frac{k_{0}^{2} p^{2}}{4 \eta^{2}}}}{\eta} d \eta\right)$

First, taking into account the approximation of the Hankel function for small arguments [59], we have:

$\lim _{\rho \rightarrow 0} H_{0}^{(2)}\left(k_{0} \rho\right) \approx 1-j \frac{2}{\pi} \ln \left(\frac{k_{0} \rho}{2}\right)-j \frac{2}{\pi} \gamma$

where $\gamma$ is the Euler constant [59]. Then, expressing the integral in (42) through the exponential integral $E_{q}(z)$ [13]:

$$
\frac{2 j}{\pi} \int_{E_{s p l}}^{\infty} \frac{e^{-\frac{\rho^{2} \eta^{2}}{p^{2}}+\frac{k_{0}^{2} p^{2}}{4 \eta^{2}}}}{\eta} d \eta=\frac{j}{\pi} \sum_{q=0}^{\infty}\left(\frac{k_{0} p}{2 E_{s p l}}\right)^{2 q} \frac{1}{q !} E_{q+1}\left(\frac{\rho^{2} E_{s p l}^{2}}{p^{2}}\right)
$$

and using the approximation of the exponential integral for small arguments [59]:

$$
\begin{aligned}
& \frac{j}{\pi} \sum_{q=0}^{\infty}\left(\frac{k_{0} p}{2 E_{s p l}}\right)^{2 q} \frac{1}{q !} E_{q+1}\left(\frac{\rho^{2} E_{s p l}^{2}}{p^{2}}\right) \approx \\
& \frac{j}{\pi}\left\{-\gamma-\ln \left(\frac{\rho^{2} E_{s p l}^{2}}{p^{2}}\right)+\sum_{q=1}^{\infty}\left(\frac{k_{0} p}{2 E_{s p l}}\right)^{2 q} \frac{1}{q q !}\right\}
\end{aligned}
$$

the expression inside the round brackets in (42) can be rewritten as:

$$
\begin{aligned}
& -1+j \frac{2}{\pi} \ln \left(\frac{k_{0} \rho}{2}\right)+j \frac{2}{\pi} \gamma+ \\
& +\frac{j}{\pi}\left[-\gamma-\ln \left(\frac{\rho^{2} E_{s p l}^{2}}{p^{2}}\right)+\sum_{q=1}^{\infty}\left(\frac{k_{0} p}{2 E_{s p l}}\right)^{2 q} \frac{1}{q q !}\right]
\end{aligned}
$$

Finally, taking into account that:

$$
j \frac{2}{\pi} \ln \left(\frac{k_{0} \rho}{2}\right)=\frac{j}{\pi} \ln \left(\frac{k_{0}^{2} \rho^{2}}{4}\right)
$$


$\frac{j}{\pi} \ln \left(\frac{k_{0}^{2} \rho^{2}}{4}\right)-\frac{j}{\pi} \ln \left(\frac{\rho^{2} E_{s p l}^{2}}{p^{2}}\right)=\frac{j}{\pi} \ln \left(\frac{k_{0}^{2} p^{2}}{4 E_{s p l}^{2}}\right)$

the $\rho$ dependence disappears and we obtain for (42) the following closed form expression:

$$
\begin{aligned}
& -1+\frac{j}{\pi}\left[\gamma+\ln \left(\frac{k_{0}^{2} p^{2}}{4 E_{s p l}^{2}}\right)+\sum_{q=1}^{\infty}\left(\frac{k_{0} p}{2 E_{s p l}}\right)^{2 q} \frac{1}{q q !}\right] \\
& =-1+\frac{j}{\pi} \operatorname{Ei}\left(\frac{k_{0}^{2} p^{2}}{4 E_{s p l}^{2}}\right)
\end{aligned}
$$

where the exponential integral (Ei) function in (49) is defined as in [59].

\section{APPENDIX C}

We evaluate here the limit expression for $\rho \rightarrow 0$ of the spectral Ewald series in (35). We make the substitution $\eta /=1 / \eta$ in the inner integral (35) and expand $\exp \left[-y^{2} /(p \eta)^{2}\right]$ into the series:

$e^{-\frac{y^{2}}{p^{2} q_{0}}}=\sum_{q=0}^{+\infty} \frac{(-1)^{q}}{q !}\left(\frac{y}{p}\right)^{2 q} \frac{1}{\eta \gamma^{q}}$

Then, using the following integral expression [66]:

$$
\begin{aligned}
& \int_{1 / E_{s p l}}^{\infty} \frac{e^{\frac{k_{y n}^{2} p^{2}}{4} \% \%}}{\eta \gamma^{q}} d \% \% \\
& =\frac{1}{2}\left(-j \frac{k_{y n} p}{2}\right)^{2 q-1} \Gamma\left(\frac{1}{2}-q,-\frac{k_{y n}^{2} p^{2}}{4 E_{s p l}^{2}}\right)
\end{aligned}
$$

where the incomplete $\Gamma$ function in (51) is defined as in [59], (35) can be cast in the following form:

$$
\begin{aligned}
& L_{m \geq 0}^{\mathrm{spec}}=\frac{j}{2 \pi^{3 / 2}} \lim _{\rho \rightarrow 0}\left\{\frac { 1 } { J _ { m } ( k _ { 0 } \rho ) } \sum _ { n = - \infty } ^ { + \infty } \left[\sum_{q=0}^{+\infty} \frac{(-1)^{q}}{q ! p^{2 q}}\right.\right. \\
& \left.\left.\times\left(-j \frac{k_{y n} p}{2}\right)^{2 q-1} \Gamma\left(\frac{1}{2}-q,-\frac{k_{y n}^{2} p^{2}}{4 E_{s p l}^{2}}\right) \int_{0}^{2 \pi} y^{2 q} e^{-j k_{x n} x} e^{j m \theta} d \theta\right]\right\}
\end{aligned}
$$

Taking into account that $y=\rho \sin \theta$ and $e^{-j k_{x n} x}=$ $\sum_{s=-\infty}^{+\infty}(-j)^{s} J_{s}\left(\left|k_{x n}\right| \rho\right) e^{-j s\left(\theta-\chi_{n}\right)}$, where $\chi_{n}$ is equal to 0 or $\pi$, the integral in (52) has the following form:

$$
\begin{aligned}
& \int_{0}^{2 \pi} y^{2 q} e^{-j k_{x n} x} e^{j m \theta} d \theta \\
& =\rho^{2 q} \sum_{s=-\infty}^{+\infty}(-j)^{s} J_{s}\left(\left|k_{x n}\right| \rho\right) e^{j s \chi_{n}} \int_{0}^{2 \pi} \sin ^{2 q}(\theta) e^{j(m-s) \theta} d \theta
\end{aligned}
$$

Since $\int_{0}^{2 \pi} \sin ^{2 q}(\theta) e^{j(m-s) \theta} d \theta=0$, when $(m-s)$ is odd and when $(m-s)$ is even, with $(m-s) \equiv 2 s^{\prime}$ for $\left|s^{\prime}\right|>q$ (see Section 3.631 in [66]), (53) can be written as:

$$
\begin{cases}\int_{0}^{2 \pi} y^{2 q} e^{-j k_{x n} x} e^{j m \theta} d \theta=\pi \rho^{2 q} \sum_{s^{\prime}=-\infty}^{+\infty}(-j)^{m-2 s^{\prime}} \frac{(-1)^{s^{\prime}}}{2^{2 q-1}} \\ \times J_{m-2 s^{\prime}}\left(\left|k_{x n}\right| \rho\right) e^{j\left(m-2 s^{\prime}\right) \chi_{n}} \frac{(2 q) !}{\left(q-s^{\prime}\right) !\left(q+s^{\prime}\right) !}, \quad q \geq\left|s^{\prime}\right| . \\ \int_{0}^{2 \pi} y^{2 q} e^{-j k_{x n} x} e^{j m \theta} d \theta=0, & q<\left|s^{\prime}\right|\end{cases}
$$

and (52) is reduced to the following expression:

$L_{m \geq 0}^{\mathrm{spec}}=-\frac{(-j)^{m+1}}{\pi^{1 / 2}} \lim _{\rho \rightarrow 0}\left\{\frac{1}{J_{m}\left(k_{0} \rho\right)} \sum_{n=-\infty}^{+\infty} \sum_{s^{\prime}=-\infty}^{+\infty}\left[\sum_{q=\left|s^{\prime}\right|}^{+\infty} \frac{(-1)^{q}}{2^{2 q}}\right.\right.$

$\times\left(\frac{\rho}{p}\right)^{2 q}\left(-j \frac{k_{y n} p}{2}\right)^{2 q-1} \Gamma\left(\frac{1}{2}-q,-\frac{k_{y n}^{2} p^{2}}{4 E_{s p l}^{2}}\right) J_{m-2 s^{\prime}}\left(\left|k_{x n}\right| \rho\right)$

$\left.\left.\times e^{j m \chi_{n}} \frac{(2 q) !}{q !\left(q-s^{\prime}\right) !\left(q+s^{\prime}\right) !}\right]\right\}$

where the simple relations $(-j)^{-2 s^{\prime}}=(-1)^{s^{\prime}}, e^{j\left(m-2 s^{\prime}\right) \chi_{n}}=e^{j m \chi_{n}}$, and $j(-j)^{m}=-(-j)^{m+1}$ have been applied.

In order to evaluate the limit $\rho \rightarrow 0$, we consider the small argument approximation of the Bessel function of the first kind of order $m$, i.e., $J_{m}(z) \approx(z / 2)^{m} / m$ !. We note that when $\rho \rightarrow 0$, the right side of (55) always becomes zero except when $0 \leq s^{\prime} \leq[m / 2]$, with $q=s^{\prime}[66]$ and

$[m / 2]=\left\{\begin{array}{l}m / 2, \quad m=0,2,4, . . \\ (m-1) / 2, \quad m=1,3,5, . .\end{array}\right.$.

This is because only for $q=s^{\prime}$, do terms with $\rho$ simplify. In all the remaining cases, $\rho$ appears in the numerator and in the limit $\rho \rightarrow 0$ the term on the right side of (55) tends to zero. As a result, (55) is rewritten as follows:

$$
\begin{aligned}
& L_{m \geq 0}^{\mathrm{spec}}=-\frac{(-j)^{m+1} m !}{\pi^{1 / 2}}\left[\sum_{n=-\infty}^{+\infty} \sum_{s^{\prime}=0}^{[m / 2]} \frac{1}{\left(k_{x n} p\right)^{2 s^{\prime}}}\left(-j \frac{k_{y n} p}{2}\right)^{2 s^{\prime}-1}\right. \\
& \left.\times \frac{(-1)^{s^{\prime}}}{s^{\prime} !\left(m-2 s^{\prime}\right) !}\left(\frac{k_{x n}}{k_{0}}\right)^{m} \Gamma\left(\frac{1}{2}-s^{\prime},-\frac{k_{y n}^{2} p^{2}}{4 E_{s p l}^{2}}\right)\right]
\end{aligned}
$$

The expression (57) can be further simplified by considering the equality $2^{2 s^{\prime}} s^{\prime} ! \pi^{1 / 2}=(-1)^{s^{\prime}} \Gamma\left(1 / 2-s^{\prime}\right)\left(2 s^{\prime}\right)$ ! and expressing the incomplete gamma function as follows: 


$$
\begin{aligned}
& \frac{\Gamma\left(\frac{1}{2}-s^{\prime},-\frac{k_{y n}^{2} p^{2}}{4 E_{s p l}^{2}}\right)}{\Gamma\left(1 / 2-s^{\prime}\right)} \\
& =\operatorname{erfc}\left(j \frac{p k_{y n}}{2 E_{s p l}}\right)-e^{\left(\frac{p k_{y n}}{2 E_{s p l}}\right)^{2}} \sum_{i=1}^{s^{\prime}} \frac{\left(j \frac{p k_{y n}}{2 E_{s p l}}\right)^{1-2 i}}{\Gamma\left(\frac{3}{2}-j\right)}
\end{aligned}
$$

The latter can be obtained if we apply a repeated integration by parts to the incomplete Gamma function [58]. Number of repetition depends on index $s^{\prime}$.

\section{REFERENCES}

[1] K. Yasumoto, Electromagnetic Theory and Applications for Photonic Crystals. Boca Raton, FL: CRC Press, 2005.

[2] B. A. Munk, Frequency Selective Surfaces: Theory and Design. New York, John Wiley \& Sons Inc., 2000.

[3] E. Yablonovitch, "Photonic band-gap structures", J. Opt. Soc. Am. B, vol. 10, n.2, pp. 283-295, 1993.

[4] F. Yang and Y. Rahmat-Samii, Electromagnetic Band Gap Structures in Antenna Engineering. Cambridge, U.K.: Cambridge Univ. Press, 2008.

[5] A. Mekis, S. Fan, and J. D. Joannopoulos, "Bound states in photonic crystal waveguides and waveguide bends," Phys. Rev. B, vol. 58, no. 8, pp. 4809-4817, Aug. 1998.

[6] G. Valerio, F. Ghasemifard, Z. Sipus, and O. Quevedo-Teruel, "Glidesymmetric all-metal holey metasurfaces for low-dispersive artificial materials: modelling and properties," IEEE Trans. Microw. Theory. Tech, in press, 2018 .

[7] S. Enoch, G. Tayeb, and D. Maystre, "Dispersion diagrams of Bloch modes applied to the design of directive sources," Progr. Electromagn. Res., vol. 41, pp. 61-81, 2003.

[8] A. R. Weily, L. Horvath, K. P. Esselle, B. C. Sanders, and T. S. Bird, "A planar resonator antenna based on a woodpile EBG material," IEEE Trans. on Antennas Propag., vol. 53, no. 1, pp. 216-223, Jan. 2005.

[9] S. Ceccuzzi, L. Pajewski, C. Ponti, and G. Schettini, "Directive EBG antennas: A comparison between two different radiating mechanisms," IEEE Trans. Antennas Propag., vol. 62, no. 10, pp. 5420-5424, Oct. 2014.

[10] R. E. Jorgenson and R. Mittra, "Efficient calculations of the free-space periodic Green's function," IEEE Trans. Antennas Propagat., vol. 38, pp. 633-642, May 1990

[11] M. E. Veysoglu, H. Yueh, R. Shin, and J. Kong, "Polarimetric passive remote sensing of periodic surfaces," J. Electromagn.Waves Appl., vol. 5, no. 3, pp. 267-280, Mar. 1991.

[12] A. W. Mathis and A. F. Peterson, "A comparison of acceleration procedures for the two-dimensional periodic Green's function," IEEE Trans. Antennas Propag., vol. 44, pp. 567-571, Apr. 1996.

[13] F. Capolino, D. R. Wilton, and W. A. Johnson, "Efficient computation of the 2-D Green's function for 1-D periodic structures using the Ewald method," IEEE Trans. Antennas Propag., vol. 53, no. 9, pp. $2977-$ 2984, Sept. 2005.

[14] G. Valerio, P. Baccarelli, P. Burghignoli, and A. Galli, "Comparative analysis of acceleration techniques for 2-D and 3-D Green's functions in periodic structures along one and two directions," IEEE Trans. Antennas Propag., vol. 55, n. 6, pp. 1630-1643, 2007.

[15] A. L. Fructos, R. R. Boix, F. Mesa, and F. Medina, "An efficient approach for the computation of 2D Green's functions with 1D and 2D periodicities in homogeneous media," IEEE Trans. Antennas Propag., vol. 56, pp. 3733-3742, Dec. 2008.

[16] D. A. Van Orden and V. Lomakin, "Rapidly convergent representations for 2D and 3D Green's functions for a linear periodic array of dipole sources," IEEE Trans. Antennas Propag., vol. 57, pp. 1973-1984, Jul. 2009.

[17] S. P. Skobelev, "A modification of the Kummer's method for efficient computation of the 2-D and 3-D Green's functions for 1-D periodic structures," IEEE Trans. Antennas Propag., vol. 60, no. 1, pp. 412-416, Jan. 2012.

[18] S. Boutami and M. Fall, "Calculation of the free-space periodic Green's function using equivalent finite array," IEEE Trans. Antennas Propag., vol. 60 , no. 10 , pp. $4725-4731$, Oct. 2012

[19] N. A. Nicorovici, R. C. McPhedran, and R. Petit, "Efficient calculation of the Green's function for electromagnetic scattering by gratings," Phys. Rev. E., vol. 49, no. 5, pp. 4563-4577, May 1994.

[20] S. K. Chin, N. A. Nicorovici, and R. C. McPhedran, "Green's function and lattice sums for electromagnetic scattering by a square array of cylinders," Phys. Rev. E, vol. 49, no. 5, pp. 4590-4602, May 1994.

[21] N. A. Nicorovici and R. C. McPhedran, "Lattice sums for off-axis electromagnetic scattering by gratings," Phys. Rev. E., vol. 50, no. 4, pp. 3143-3160, Oct. 1994.

[22] C. M. Linton, "The Green's function for the two-dimensional Helmholtz equation in periodic domains," J. Eng. Math., vol. 33, no. 4, pp. 377402, May 1998.

[23] K. Yasumoto and K. Yoshitomi, "Efficient calculation of lattice sums for free-space periodic Green's function," IEEE Trans. Antennas Propag., vol. 47, pp. 1050-1055, June 1999.

[24] A. Lakhtakia, V. V. Varadan, and V. K. Varadan, "Reflection characteristics of a dielectric slab containing dielectric or perfectly conducting cylindrical gratings," Appl. Opt., vol. 25, no. 6, pp. 887894, Mar. 1986

[25] K. Yasumoto, H. Toyama, and R. Kushta, "Accurate analysis of twodimensional electromagnetic scattering from multilayered periodic arrays of circular cylinders using lattice sums technique," IEEE Trans. Antennas Propag., vol. 52, no. 10, pp. 2603-2611, Oct 2004.

[26] J. Yang, L. W. Li, K. Yasumoto, and C. H. Liang, "Two-dimensional scattering of a gaussian beam by a periodic array of circular cylinders," IEEE Trans. Geosci. Remote Sens., vol. 43, no. 2, pp. 280-285, Feb. 2005

[27] K. Yasumoto, H. Jia, and K. Sun, "Rigorous modal analysis of twodimensional photonic crystal waveguide," Radio Sci., vol. 40, no. 6 (RS6S02), pp. 1-7, Sep. 2005.

[28] L. C. Botten, N. A. Nicorovici, A. Asatryan, R. C. McPhedran, C. M. de Sterke and P. A. Robinson, "Formulation for electromagnetic scattering and propagation through grating stacks of metallic and dielectric cylinders for photonic crystal calculations. Part I. Method," J. Opt. Soc. Amer. A, vol. 17, pp. 2165-2176, 2000.

[29] S. Wilcox, L. C. Botten, and R. C. McPhedran, "Modeling of defect modes in photonic crystals using the fictitious source superposition method," Phys. Rev. E, vol. 71, pp. 1-11, 2005.

[30] C. Li, D. Lesselier, and Y. Zhong, "Electromagnetic small-scale modeling of composite panels involving periodic arrays of circular fibers," Appl. Phys. A, vol. 117, pp. 567-572, 2014.

[31] C. Y. Li, D. Lesselier, and Y. Zhong, "Scattering of obliquely incident electromagnetic plane waves by composite panel involving periodic arrays of circular fibers," IEEE Trans. Antennas Propag., vol. 63, no. 7, pp. 3168-3178, Jul. 2015.

[32] C. Li, D. Lesselier, and Y. Zhong, "Full-wave computational model of electromagnetic scattering by arbitrarily rotated 1-D periodic multilayer structure," IEEE Trans. Antennas Propag., vol. 64, no. 3, pp. 10471060, Mar. 2016.

[33] G. N. Watson, A Treatise on the Theory of Bessel Functions. Cambridge, U.K.: Cambridge Univ. Press, 1962, ch. 19.

[34] V. Twersky, "Elementary function representations of Schlömilch series," Arch. Ration. Mech. Anal., vol. 8, pp. 323-332, 1961.

[35] C. M. Linton, "Schlömilch series that arise in diffraction theory and their efficient computation," J. Phys. A: Math. Gen., vol. 39, pp. 25-33, 2006

[36] A. Moroz, "Exponentially convergent lattice sums," Opt. Lett., vol. 26, pp. 1119-1121, 2001.

[37] C. M. Linton and I. Thompson, "One- and two-dimensional lattice sums for the three-dimensional Helmholtz equation," J. Comput. Phys., vol. 228, pp. 1815-1829, Apr. 2009.

[38] C. M. Linton, "Lattice sums for the Helmholtz equation," SIAM Rev., vol. 52, no. 4, pp. 630-674, 2010.

[39] S. Ceccuzzi, V. Jandieri, P. Baccarelli, C. Ponti, and G. Schettini, "On beam shaping of the field radiated by a line source coupled to finite or infinite photonic crystals," J. Opt. Soc. Amer. A, vol. 33, n. 4, pp. 764770, Apr. 2016. 
[40] S. Ceccuzzi, C. Ponti, and G. Schettini, "Directive EBG antennas based on lattice modes", IEEE Trans. Antennas Propag., vol. 65, n. 4, pp. 1691-1699, Apr. 2017.

[41] D. R. Jackson and A. A. Oliner, "Leaky-wave antennas", in Balanis, C.A. (ed.), Modern Antenna Handbook, Wiley, New York, NY, 2008, Ch. 7.

[42] G. Valerio et al., "Efficient computation of 1-D periodic layered mixed potentials for the analysis of leaky-wave antennas with vertical elements," IEEE Trans. Antennas Propag., vol. 63, no. 6, pp. 23962411, Jun. 2015

[43] D. Deslandes and K. Wu, "Accurate modeling, wave mechanisms, and design considerations of a substrate integrated waveguide," IEEE Trans. Microwave Theory Tech., vol. 54, n. 6, pp. 2516-2526, 2006.

[44] V. Jandieri, H. Maeda, K. Yasumoto and D. Erni, "Analysis of post-wall waveguides and circuits using a model of two-dimensional photonic crystals," Progress in Electromagnetics Research M (PIER M), vol. 56 pp. 91-100, 2017.

[45] R. Qiang, J. Chen, F. Capolino, D. R. Jackson, and D. R. Wilton, "ASM-FDTD: A technique for calculating the field of a finite source in the presence of an infinite periodic artificial material," IEEE Microw. Wireless Compon. Lett., vol. 17, no. 4, pp. 271-273, Apr. 2007.

[46] S. Paulotto, G. Lovat, P. Baccarelli, and P. Burghignoli, "Accurate Green's function calculation for a line source exciting a 2-D periodic printed structure," IEEE Microw. Wireless Compon. Lett., vol. 20, no. 4, pp. 181-183, Apr. 2010.

[47] S. Paulotto, P. Baccarelli, P. Burghignoli, G. Lovat, G. Hanson, and A. B. Yakovlev, "Homogenized Green's functions for an aperiodic line source over planar densely periodic artificial impedance surfaces," IEEE Trans. Microwave Theory Tech., vol. 58, no. 7, pp. 1807-1817, Jul. 2010

[48] P.P. Ewald, "Die Berechnung optischer und elektrostatischer Gitterpotentiale," Ann. der Physik, vol. 369, n. 3, pp. 253-287, 1921.

[49] P. Baccarelli, V. Jandieri, G. Valerio, and G. Schettini, "Efficient computation of the lattice sums for leaky waves using the Ewald method," 11-th European Conference on Antennas and Propagation, Paris, France, 19-24 March, 2017, pp. 3222-3223.

[50] V. Jandieri, P. Baccarelli, C. Ponti, and G. Schettini, "Full-wave analysis of leaky modes in 2-D EBG waveguides," 11-th European Conference on Antennas and Propagation, Paris, France, 19-24 March, 2017, pp. 3224-3225.

[51] V. Jandieri, P. Baccarelli, G. Valerio, S. Ceccuzzi, C. Ponti, G. Schettini, "Efficient and rigorous analysis of leaky modes in 2-D EBG guiding structures," 19-th International Conference on Electromagnetics in Advanced Applications, Verona, Italy, 11-15 September, 2017, pp. 444-445.

[52] W. A. Johnson, L. I. Basilio, J. D. Kotulski, R. E. Jorgenson, L. K. Warne, R. S. Coats, D. R. Wilton, N. J. Champagne, F. Capolino, J. B. Grant, and M. A. Khayat, "EIGER: An open-source frequency-domain electromagnetics code," in Proc. IEEE Int. Symp. on Antennas and Propagation, Honolulu, HI, Jun. 2007, pp. 3328-3331.

[53] G. Valerio, A. Galli, D. R. Wilton, and D. R. Jackson, "An enhanced integral-equation formulation for accurate analysis of frequencyselective structures," Int. J. Microwave Wireless Techn., vol. 4, no. 3, pp. 365-372, 2012.

[54] E. W. Lucas and T. W. Fontana, "A 3-D hybrid finite element/boundary element method for the unified radiation and scattering analysis of general infinite periodic arrays," IEEE Trans. Antennas Propagat., vol. 43, no. 2, pp. 145-153, Feb. 1995.

[55] T. F. Eibert, Y. Weitsch, H. Chen and M. E. Gruber, "Solving periodic eigenproblems by solving corresponding excitation problems", Progress in Electromagnetics Research, vol. 126, pp. 65-84, 2012.

[56] S. Tooni and T. F. Eibert, "Excitation of complex modes in periodic structures using inhomogeneous plane wave scattering in fast and slow wave regions, IEEE Trans. Antennas Propag., vol. 62, no. 12, pp. 6290-6298, Dec. 2014.

[57] D. Zhang and H. Jia, "Numerical analysis of leaky modes in twodimensional photonic crystal waveguides using Fourier series expansion method with perfectly matched layer," IEICE Trans. Electron., E90-C, pp. 613-622, 2007

[58] S. Li and Y. Y. Lu, "Efficient method for computing leaky modes in two-dimensional photonic crystal waveguides," J. Lightw. Technol., vol. 28, n. 6, pp.978-983, Mar. 2010.
[59] M. Abramowitz and I. A. Stegun, Handbook of Mathematical Functions, Dover, New York, NY, USA, 1962.

[60] V. Jandieri and K. Yasumoto, "Electromagnetic Scattering by Layered Cylindrical Arrays of Circular Rods," IEEE Trans. Antennas Propag., vol.59, n.6, pp. 2437-2441, Jun. 2011.

[61] V. Jandieri, K. Yasumoto and B. Gupta, "Directivity of radiation from a localized source coupled to electromagnetic crystals", Journal of Infrared, Millimetre and Terahertz Waves, vol. 30, n. 10, pp. 11021112,2009

[62] A. Hochman and Y. Leviatan, "Rigorous modal analysis of metallic nanowire chains," Optics Express, vol. 17, n. 16, pp. 13561-13575, 2009.

[63] H. Jia, K. Yasumoto, and H. Toyama, "Reflection and transmission properties of layered periodic arrays of circular cylinders embedded in magnetized ferrite slab," IEEE Trans. Antennas Propag., vol. 53, no. 3 , pp. 1145-1153, Mar. 2005.

[64] D. Felbacq, G. Tayeb and D. Maystre, "Scattering by a random set of parallel cylinders," J. Opt. Soc. Amer. A, vol. 11, n. 9, pp. 2526-2538, Sep. 1994.

[65] F. T. Celepcikay, F. Capolino, D. R. Jackson, and D. R. Wilton, "Choosing splitting parameters and summation limits in the numerical evaluation of 1-D and 2-D periodic Green's functions using the Ewald method," Radio Science, vol. 43, RS6S01, 2008.

[66] I. Gradshteyn and I. Ryzhik, Table of Integrals, Series, and Products, 6th ed., New York, NY, USA: Academic, 2000.

[67] K. E. Atkinson, An introduction to numerical analysis, $2^{\text {nd }}$ ed., Wiley, New York, NY, 1989.

[68] K. Kambe, "Theory of Low-Energy Electron Diffraction," Zeitschrift für Naturforschung, vol. 22a, pp. 322-330, 1967.

[69] T. Arens, K. Sandfort, S. Schmitt, and A. Lechleiter, "Analysing Ewald's method for the evaluation of Green's functions for periodic media," IMA J. Appl. Math., vol. 78, no. 3, pp. 405-431, 2013.

[70] H. S. Carslaw, Introduction to the Theory of Fourier's Series and Integrals, 3rd ed. New York: Dover Publications Inc., 1930.

[71] F. T. Celepcikay, D. R. Wilton, D. R. Jackson, W. A. Johnson, "Interpolation of Ewald-accelerated periodic Green's function representations for homogeneous or layered media," IEEE Trans. Antennas and Propagat., vol. 65, n. 5, pp. 2517-2525, May 2017.

[72] G. Valerio, P. Baccarelli, S. Paulotto, F. Frezza, and A. Galli, "Regularization of mixed-potential layered-media Green's functions for efficient interpolation procedures in planar periodic structures," IEEE Trans. Antennas Propag., vol. 57, no. 1, pp. 122-134, Jan. 2009.

[73] P. Baccarelli, S. Paulotto, and C. Di Nallo, "Full-wave analysis of bound and leaky modes propagating along $2 \mathrm{D}$ periodic printed structures with arbitrary metallisation in the unit cell," IET Microw. Antennas Propag., Special Issue on Metamaterials EBG, vol. 1, n. 1, 217-225, 2007. 